\title{
Anisotropic optical trapping as a manifestation of the complex electronic structure of ultracold lanthanide atoms: The example of holmium
}

\author{
Hui Li, ${ }^{1}$ Jean-François Wyart, ${ }^{1,2}$ Olivier Dulieu, ${ }^{1}$ and Maxence Lepers ${ }^{1, *}$ \\ ${ }^{1}$ Laboratoire Aimé Cotton, CNRS, Université Paris-Sud, ENS Paris-Saclay, Université Paris-Saclay, 91405 Orsay, France \\ ${ }^{2}$ LERMA, Observatoire de Paris-Meudon, PSL Research University, Sorbonne Universités, \\ UPMC Université Paris 6, CNRS UMR8112, 92195 Meudon, France
}

(Received 13 April 2017; published 26 June 2017)

\begin{abstract}
The efficiency of optical trapping is determined by the atomic dynamic dipole polarizability, whose real and imaginary parts are associated with the potential energy and photon-scattering rate, respectively. In this article we develop a formalism to calculate analytically the real and imaginary parts of the scalar, vector, and tensor polarizabilities of lanthanide atoms. We assume that the sum-over-state formula comprises only transitions involving electrons in the valence orbitals like $6 s, 5 d, 6 p$, and $7 s$, while transitions involving $4 f$ core electrons are neglected. Applying this formalism to the ground level of configuration $4 f^{q} 6 s^{2}$, we restrict the sum to transitions implying the $4 f^{q} 6 s 6 p$ configuration, which yields polarizabilities depending on two parameters: an effective transition energy and an effective transition dipole moment. Then, by introducing configuration-interaction mixing between $4 f^{q} 6 s 6 p$ and other configurations, we demonstrate that the imaginary part of the scalar, vector, and tensor polarizabilities is very sensitive to configuration-interaction coefficients, whereas the real part is not. The magnitude and anisotropy of the photon-scattering rate are thus strongly related to the details of the atomic electronic structure. Those analytical results agree with our detailed electronic-structure calculations of the energy levels, Landé $g$ factors, transition probabilities, polarizabilities, and van der Waals $C_{6}$ coefficients, previously performed on erbium and dysprosium and presently performed on holmium. Our results show that, although the density of states decreases with increasing $q$, the configuration interaction between $4 f^{q} 6 s 6 p, 4 f^{q-1} 5 d 6 s^{2}$, and $4 f^{q-1} 5 d^{2} 6 s$ is surprisingly stronger in erbium $(q=12)$ than in holmium $(q=11)$, itself stronger than in dysprosium $(q=10)$.
\end{abstract}

DOI: 10.1103/PhysRevA.95.062508

\section{INTRODUCTION}

The physics of ultracold gases has evolved rapidly and is poised to enter a new, promising regime, where complex atomic and molecular species can be cooled and studied extensively. Lanthanide atoms, with a strong magnetic moment and a large orbital angular momentum, are extreme examples of such complex species. In fact, the interest in ultracold lanthanide atoms is motivated by several topics in current research, including ultracold collisions and quantum chaos [1-3], dipolar quantum gases with large magnetic moments and strong dipole-dipole interactions [4-8], many-body quantum systems $[9,10]$, exotic quantum phases [11-13] like stable quantum droplets [14-16], synthetic gauge fields [17,18], and optical clocks [19-21]. Recent progress in laser cooling and magneto-optical trapping of high-atomic-number (high$Z$ ) lanthanides [22,23], including dysprosium (Dy) [24-27], erbium (Er) [28-30], holmium (Ho) [31], and thulium (Tm) [32] is paving the way towards these investigations. In addition, both Bose-Einstein condensates and quantum-degenerate Fermi gases have been produced in isotopes of Dy [4,33,34] and $\operatorname{Er}[35,36]$.

The ground level of holmium is characterized by the electronic configuration [Xe] $4 f^{11} 6 s^{2}$ and electronic angular momentum $J=15 / 2$. Due to the nuclear spin $I=7 / 2$ of its only stable (bosonic) isotope ${ }^{165} \mathrm{Ho}$, holmium is the atom possessing the largest number of hyperfine sublevels in the electronic ground level, namely, $(2 J+1) \times(2 I+1)=128$.

\footnotetext{
*maxence.lepers@u-psud.fr
}

This rich structure is likely to be exploited in quantum information [37,38]. Like other lanthanides, the complex electronic structure of holmium induces a large magnetic dipole moment $\left(9 \mu_{B}\right)$, which makes it an interesting candidate for the investigation of anisotropic interactions between atoms $[39,40]$. Recently the holmium single magnetic atom and holmium molecular nanomagnet were also presented as competing candidates for the realization of quantum bits [41,42].

Many of the applications listed above involve optically trapped ultracold atoms. The trapping efficiency is determined by the interaction between the atoms and the electromagnetic field $[43,44]$. The microscopic property characterizing the atomic response is the (complex) dynamic dipole polarizability (DDP). On the one hand, the field induces a potential energy, i.e., an ac-Stark shift, in the atoms, which is proportional to the real part of the DDP. On the other hand, the field also induces photon scattering, whose rate is proportional to the imaginary part of the DDP. In ultracold experiments, it is necessary to characterize the photon-scattering rate, as it provokes heating of the sample and trap losses [44]. Beyond trapping itself, the real part of the vector and tensor DDPs is also necessary to determine the Raman coupling strengths between different Zeeman sublevels, which was proposed for the implementation of synthetic gauge fields [17,18]. In our previous works on Er [45] and Dy [46], we have shown that, far from resonant frequencies, the ac-Stark shift only weakly depends on the field polarization and atomic Zeeman sublevel, despite the absence of spherical symmetry in the $4 f$-electron wave functions. We have revealed the inverse situation for photon scattering, as the imaginary part of the vector and tensor DDPs represents significant fractions of the scalar one. 
This opens the possibility of controlling the trap heating and losses with an appropriate field polarization. However, the vector-to-scalar and tensor-to-scalar ratios vary strongly from Dy to Er, which is still unexplained.

Understanding the origin of that difference is a major motivation of the present work. Moreover, ultracold experiments may require characterization of the optical trapping of atomic excited levels with energies up to $25000 \mathrm{~cm}^{-1}$ above ground level. Calculating the DDP of such levels with the sum-over-state formula requires modeling highly excited levels, roughly up to $60000 \mathrm{~cm}^{-1}$ above ground level, which is a hard task for the most complex spectra of lanthanide atoms. Therefore, in this article, we present a simplified model of the DDP based on the sum-over-state formula, where we suppose that the only contributions come from transitions involving valence electrons like $6 s, 6 p, 5 d$, and $7 s$ and where we ignore transitions involving $4 f$ core electrons. Assuming that all the levels of a given configuration have similar energies, we obtain analytical expressions of the DDPs of an arbitrary level, depending on a restricted number of effective parameters. Focusing on the ground level of the configuration [Xe] $4 f^{q} 6 s^{2}$ ( $q=10,11$, and 12 for Dy, Ho, and Er, respectively), we take into account only the excitation from the $6 s$ to the $6 p$ orbital, and not the excitation from the $4 f$ to the $5 d$ orbital. We demonstrate that the real part of the DDP is not influenced by the configuration interaction $(\mathrm{CI})$ between $[\mathrm{Xe}] 4 f^{q} 6 s 6 p$ and other configurations like [Xe] $4 f^{q-1} 5 d 6 s^{2}$ and $[\mathrm{Xe}] 4 f^{q-1} 5 d^{2} 6 s$. Our model also shows that the real part of the vector and tensor ground-level DDPs vanish. By contrast, the imaginary part of the DDPs is very sensitive to CI, in particular, to the weight of the $[\mathrm{Xe}] 4 f^{q} 6 s 6 p$ configuration in excited levels. We demonstrate that strong CI mixing tends to increase the vector and tensor DDPs with respect to the scalar one. Surprisingly, CI mixing turns out to be larger for Er than for Ho, and for Ho than for Dy, although the energy spectrum of Dy is the densest one.

In order to check the validity of those conclusions, we perform a full numerical modeling of holmium spectrum, including energy levels, transition probabilities, polarizabilities, and van der Waals $C_{6}$ coefficients, complementing our previous studies on erbium [45] and dysprosium [46]. The DDPs and $C_{6}$ coefficients are calculated using the sum formula involving transition energies and transition dipole moments extracted from our computed transition probabilities. Following our previous work [45-48], those quantities are calculated using a combination of $a b$ initio and least-squares fitting procedures provided by the Cowan suite of codes [49] and extended in our group. Therefore we provide a theoretical interpretation of Ho even-parity levels, which especially results in the prediction of the widely unmeasured Landé $g$ factors. Because the spectrum of high- $Z$ lanthanide atoms in the ground level is composed of a few strong transitions emerging from a forest of weak ones, the sum-over-state formula is appropriate for calculation of DDPs and $C_{6}$ coefficients. It offers the possibility of precisely calculating, with a single set of spectroscopic data, the real and imaginary parts of the scalar, vector, and tensor DDPs in a wide range of wavelengths, especially at $1064 \mathrm{~nm}$, widely used experimentally for trapping purposes.

This article is outlined as follows. We develop our simplified model for the DDP in Sec. II: we first recall useful formulas and, especially, the relationships between scalar, vector, and tensor DDPs and tensor operators (see Sec. II A). Then we calculate the contribution from the levels of a single configuration (see Sec. II B) to the real and imaginary parts of the DDPs, while the two next subsections are devoted to the influence of CI mixing in the DDPs of ground-level lanthanide atoms. Section III deals with the full numerical modeling of the holmium spectrum-energy levels, transition probabilities, polarizabilities, and van der Waals $C_{6}$ coefficients (see Secs. III A-III D, respectively). Section IV contains concluding remarks.

\section{DYNAMIC DIPOLE POLARIZABILITY: A SIMPLIFIED MODEL}

\section{A. Polarizability and tensor operators}

For non-spherically symmetric atoms like lanthanides, the ac-Stark shift is a linear combination of three terms, depending on the scalar, vector, and tensor polarizabilities, taken at the angular frequency $\omega$ of the oscillating electric field (hereafter denoted the "frequency"). The magnitude of each term is a function of the atomic Zeeman sublevel $M$ and of the electric-field polarization [43]. The scalar $\alpha_{\text {scal }}(\omega)$, vector $\alpha_{\text {vect }}(\omega)$, and tensor polarizabilities $\alpha_{\text {tens }}(\omega)$ can be associated with the coupled polarizabilities $\alpha_{k}(\omega)$, where $k=0,1$, and 2 , respectively, are the ranks of the corresponding irreducible tensor [43,50]. Namely,

$$
\begin{gathered}
\alpha_{\text {scal }}(\omega)=-\frac{\alpha_{0}(\omega)}{\sqrt{3(2 J+1)}}, \\
\alpha_{\text {vect }}(\omega)=\alpha_{1}(\omega) \sqrt{\frac{2 J}{(J+1)(2 J+1)}}, \\
\alpha_{\text {tens }}(\omega)=\alpha_{2}(\omega) \sqrt{\frac{2 J(2 J-1)}{3(J+1)(2 J+1)(2 J+3)}} .
\end{gathered}
$$

For an atomic level $|\beta J\rangle$, where $J$ is the electronic-angularmomentum quantum number and $\beta$ stands for all the other quantum numbers, we write the complex polarizability $\alpha_{k}(\omega)$ by applying the sum-over-state formulation of the secondorder time-dependent perturbation theory [51] and assuming complex energies for the intermediate levels $\left|\beta^{\prime \prime} J^{\prime \prime}\right\rangle$,

$$
\begin{aligned}
\alpha_{k}(\omega)= & \sqrt{2 k+1} \sum_{\beta^{\prime \prime} J^{\prime \prime}}(-1)^{J+J^{\prime \prime}} \\
& \times\left\{\begin{array}{ccc}
1 & 1 & k \\
J & J & J^{\prime \prime}
\end{array}\right\}\left|\left\langle\beta^{\prime \prime} J^{\prime \prime}\|\mathbf{d}\| \beta J\right\rangle\right|^{2} \\
& \times\left(\frac{(-1)^{k}}{E_{\beta^{\prime \prime} J^{\prime \prime}}-E_{\beta J}-i \frac{\hbar \gamma_{\beta^{\prime \prime} J^{\prime \prime}}}{2}-\hbar \omega}\right. \\
& \left.+\frac{1}{E_{\beta^{\prime \prime} J^{\prime \prime}}-E_{\beta J}-i \frac{\hbar \gamma_{\beta^{\prime \prime} J^{\prime \prime}}}{2}+\hbar \omega}\right),
\end{aligned}
$$

where $E_{\beta J}\left(E_{\beta^{\prime \prime} J^{\prime \prime}}\right)$ are the energies of the levels $|\beta J\rangle\left(\left|\beta^{\prime \prime} J^{\prime \prime}\right\rangle\right)$, $\left\langle\beta^{\prime \prime} J^{\prime \prime}\|\mathbf{d}\| \beta J\right\rangle$ is the reduced transition dipole moment between these two levels, $\gamma_{\beta^{\prime \prime} J^{\prime \prime}}$ is the natural line width of the intermediate level $\left|\beta^{\prime \prime} J^{\prime \prime}\right\rangle$, and the quantity in curly brackets is a Wigner 6-j symbol [52]. 
We consider frequencies far from any atomic resonances, i.e., $E_{\beta^{\prime \prime} J^{\prime \prime}}-E_{\beta J} \pm \hbar \omega \gg \hbar \gamma_{\beta^{\prime \prime} J^{\prime \prime}} / 2$, which is relevant for trapping purposes and which greatly simplifies Eq. (4). We separate the real $\operatorname{Re}\left[\alpha_{k}(\omega)\right]$ and imaginary parts $\operatorname{Im}\left[\alpha_{k}(\omega)\right]$,

$$
\begin{aligned}
& \operatorname{Re}\left[\alpha_{k}(\omega)\right]=2 \sqrt{2 k+1} \sum_{\beta^{\prime \prime} J^{\prime \prime}}(-1)^{J+J^{\prime \prime}}\left\{\begin{array}{ccc}
1 & 1 & k \\
J & J & J^{\prime \prime}
\end{array}\right\}\left|\left\langle\beta^{\prime \prime} J^{\prime \prime}\|\mathbf{d}\| \beta J\right\rangle\right|^{2} \frac{\left(E_{\beta^{\prime \prime} J^{\prime \prime}}-E_{\beta J}\right) \delta_{(-1)^{k}, 1}-\hbar \omega \delta_{(-1)^{k},-1}}{\left(E_{\beta^{\prime \prime} J^{\prime \prime}}-E_{\beta J}\right)^{2}-\hbar^{2} \omega^{2}}, \\
& \operatorname{Im}\left[\alpha_{k}(\omega)\right]= \sqrt{2 k+1} \sum_{\beta^{\prime \prime} J^{\prime \prime}}(-1)^{J+J^{\prime \prime}}\left\{\begin{array}{ccc}
1 & 1 & k \\
J & J & J^{\prime \prime}
\end{array}\right\} \hbar \gamma_{\beta^{\prime \prime} J^{\prime \prime}}\left|\left\langle\beta^{\prime \prime} J^{\prime \prime}\|\mathbf{d}\| \beta J\right\rangle\right|^{2} \\
& \times \frac{\left[\left(E_{\beta^{\prime \prime} J^{\prime \prime}}-E_{\beta J}\right)^{2}+\hbar^{2} \omega^{2}\right] \delta_{(-1)^{k}, 1}-2 \hbar \omega\left(E_{\beta^{\prime \prime} J^{\prime \prime}}-E_{\beta J}\right) \delta_{(-1)^{k},-1}}{\left[\left(E_{\beta^{\prime \prime} J^{\prime \prime}}-E_{\beta J}\right)^{2}-\hbar^{2} \omega^{2}\right]^{2}}
\end{aligned}
$$

where we have used $A+(-1)^{k} B=(A+B) \delta_{(-1)^{k}, 1}+(A-B) \delta_{(-1)^{k},-1}$. Plugging Eqs. (5) and (6) into Eqs. (1)-(3) and introducing the explicit expressions of 6-j symbols (see Ref. [52], p. 302), we get to the real and imaginary parts of the scalar, vector, and tensor contributions:

$$
\begin{gathered}
\operatorname{Re}\left[\alpha_{\text {scal }}(\omega)\right]=\frac{2}{3(2 J+1)} \sum_{\beta^{\prime \prime} J^{\prime \prime}} \frac{\left(E_{\beta^{\prime \prime} J^{\prime \prime}}-E_{\beta J}\right)\left|\left\langle\beta^{\prime \prime} J^{\prime \prime}\|\mathbf{d}\| \beta J\right\rangle\right|^{2}}{\left(E_{\beta^{\prime \prime} J^{\prime \prime}}-E_{\beta J}\right)^{2}-\hbar^{2} \omega^{2}}, \\
\operatorname{Im}\left[\alpha_{\text {scal }}(\omega)\right]=\frac{1}{3(2 J+1)} \sum_{\beta^{\prime \prime} J^{\prime \prime}} \frac{\hbar \gamma_{\beta^{\prime \prime} J^{\prime \prime}}\left[\left(E_{\beta^{\prime \prime} J^{\prime \prime}}-E_{\beta J}\right)^{2}+\hbar^{2} \omega^{2}\right]\left|\left\langle\beta^{\prime \prime} J^{\prime \prime}\|\mathbf{d}\| \beta J\right\rangle\right|^{2}}{\left[\left(E_{\beta^{\prime \prime} J^{\prime \prime}}-E_{\beta J}\right)^{2}-\hbar^{2} \omega^{2}\right]^{2}}, \\
\operatorname{Re}\left[\alpha_{\text {vect }}(\omega)\right]=\sum_{\beta^{\prime \prime} J^{\prime \prime}} \frac{J^{\prime \prime}\left(J^{\prime \prime}+1\right)-J(J+1)-2}{(J+1)(2 J+1)} \times \frac{\hbar \omega\left|\left\langle\beta^{\prime \prime} J^{\prime \prime}\|\mathbf{d}\| \beta J\right\rangle\right|^{2}}{\left(E_{\beta^{\prime \prime} J^{\prime \prime}}-E_{\beta J}\right)^{2}-\hbar^{2} \omega^{2}}, \\
\operatorname{Im}\left[\alpha_{\text {vect }}(\omega)\right]=\sum_{\beta^{\prime \prime} J^{\prime \prime}} \frac{J^{\prime \prime}\left(J^{\prime \prime}+1\right)-J(J+1)-2}{(J+1)(2 J+1)} \times \frac{\hbar^{2} \omega \gamma_{\beta^{\prime \prime} J^{\prime \prime}}\left(E_{\beta^{\prime \prime} J^{\prime \prime}}-E_{\beta J}\right)\left|\left\langle\beta^{\prime \prime} J^{\prime \prime}\|\mathbf{d}\| \beta J\right\rangle\right|^{2}}{\left[\left(E_{\beta^{\prime \prime} J^{\prime \prime}}-E_{\beta J}\right)^{2}-\hbar^{2} \omega^{2}\right]^{2}}, \\
\operatorname{Re}\left[\alpha_{\text {tens }}(\omega)\right]=-\sum_{\beta^{\prime \prime} J^{\prime \prime}} \frac{3\left[J^{\prime \prime}\left(J^{\prime \prime}+1\right)-J(J+1)\right]^{2}-9 J^{\prime \prime}\left(J^{\prime \prime}+1\right)+J(J+1)+6}{3(J+1)(2 J+1)(2 J+3)} \times \frac{\left(E_{\beta^{\prime \prime} J^{\prime \prime}}-E_{\beta J}\right)\left|\left\langle\beta^{\prime \prime} J^{\prime \prime}\|\mathbf{d}\| \beta J\right\rangle\right|^{2}}{\left(E_{\beta^{\prime \prime} J^{\prime \prime}}-E_{\beta J}\right)^{2}-\hbar^{2} \omega^{2}}, \\
\operatorname{Im}\left[\alpha_{\text {tens }}(\omega)\right]=-\sum_{\beta^{\prime \prime} J^{\prime \prime}} \frac{3\left[J^{\prime \prime}\left(J^{\prime \prime}+1\right)-J(J+1)\right]^{2}-9 J^{\prime \prime}\left(J^{\prime \prime}+1\right)+J(J+1)+6}{6(J+1)(2 J+1)(2 J+3)} \\
\times \frac{\hbar \gamma_{\beta^{\prime \prime} J^{\prime \prime}}\left[\left(E_{\beta^{\prime \prime} J^{\prime \prime}}-E_{\beta J}\right)^{2}+\hbar^{2} \omega^{2}\right]\left|\left\langle\beta^{\prime \prime} J^{\prime \prime}\|\mathbf{d}\| \beta J\right\rangle\right|^{2}}{\left[\left(E_{\beta^{\prime \prime} J^{\prime \prime}}-E_{\beta J}\right)^{2}-\hbar^{2} \omega^{2}\right]^{2}}
\end{gathered}
$$

Note that in Eqs. (7), (8), and (11) of Ref. [45], the sign of the vector polarizability is not correct; the error has been fixed in Eqs. (9) and (10) above.

\section{B. Effect of a single intermediate configuration}

In this subsection, we assume that the intermediate levels $\left|\beta^{\prime \prime} J^{\prime \prime}\right\rangle$ appearing in Eq. (4) all belong to the same configuration and that their transition energies $E_{\beta^{\prime \prime} J^{\prime \prime}}-E_{\beta J}$ can be replaced by a single effective one. Moreover, we assume that the configurations of the $|\beta J\rangle$ and $\left|\beta^{\prime \prime} J^{\prime \prime}\right\rangle$ levels differ by the hopping of only one valence electron; in other words, we ignore transitions involving the $4 f$ core electrons. This will yield analytical expressions useful for estimating $\alpha_{k}(\omega)$ and understanding the trapping in some relevant levels, like those belonging to the lowest or the $[\mathrm{Xe}] 4 f^{q} 6 s 6 p$ configurations.

Many levels of lanthanide atoms can be interpreted in the frame of the $j j$ coupling scheme. The electronic core, containing the $4 f$ shell, is characterized by its orbital $L_{c}$, spin $S_{c}$, and total electronic angular momentum $J_{c}$. The valence electrons belong, for instance, to the $5 d, 6 s$, or $6 p$ shell. This group of electrons is characterized by their orbital $L_{v}$, spin $S_{v}$, and total electronic angular momentum $J_{v}$. Then $J_{c}$ and $J_{v}$ are coupled to give the total electronic angular momentum $J$ of the atomic level. In the present study, we focus on the configurations $[\mathrm{Xe}] 4 f^{q} \cdot n_{1} \ell_{1} n_{2} \ell_{2}(q=10,11$, and 12 for Dy, Ho, and Er, respectively) with two valence electrons, including, e.g., $4 f^{q} 6 s^{2}$ or $4 f^{q} 6 s 6 p$; but our results can be extended to configurations with three valence electrons like $4 f^{q-1} 5 d 6 s^{2}$ and $4 f^{q-1} 5 d^{2} 6 s$. The full label of the level is therefore $[\mathrm{Xe}] 4 f^{q}\left({ }^{2 S_{c}+1} L_{c J_{c}}\right) \cdot n_{1} \ell_{1} n_{2} \ell_{2}\left({ }^{2 S_{v}+1} L_{v J_{v}}\right)\left(J_{c}, J_{v}\right)_{J}$, and its electronic parity is $(-1)^{q+\ell_{1}+\ell_{2}}$. In what follows, we omit the xenon core $[\mathrm{Xe}]$ in electronic configurations.

It is noteworthy that the levels of the $4 f^{q} 5 d 6 s$ configuration are better described in the $j K$ coupling scheme ${ }^{2 S_{v}+1}[K]_{J}: J_{c}$ is first coupled with $L_{v}$ to give $K$, which is itself coupled with $S_{v}$ to give $J$. In order to calculate the polarizability of such levels, it is necessary to apply the basis transformation from $j j$ to $j K$ coupling schemes [49]. However, if those levels appear in the sum over $\left|\beta^{\prime \prime} J^{\prime \prime}\right\rangle$, the $j j$ coupling scheme is sufficient, as all the levels of the $4 f^{q} 5 d 6 s$ configuration are assumed to have the same energy (see Sec. II B 2).

\section{Transition dipole moment in $j j$ coupling}

In the electric-dipole (E1) approximation, the transitions with the strongest dipole moments are those for which one 
valence electron, say $n_{2} \ell_{2}$, is promoted to an orbital $n_{2}^{\prime \prime} \ell_{2}^{\prime \prime}$ such that $\ell_{2}^{\prime \prime}=\ell_{2} \pm 1$. The angular momenta of the atom must also satisfy the selection rules, $L_{v}^{\prime \prime}=L_{v}$ or $L_{v} \pm 1, S_{v}^{\prime \prime}=S_{v}$, $J_{v}^{\prime \prime}=J_{v}$ or $J_{v} \pm 1$, and $J^{\prime \prime}=J$ or $J \pm 1$, excluding transitions between couples of angular momenta $(0,0)$, whereas the quantum numbers of the core are not modified $\left(L_{c}^{\prime \prime}=L_{c}\right.$, $S_{c}^{\prime \prime}=S_{c}$, and $J_{c}^{\prime \prime}=J_{c}$ ). In the frame of the $j j$ coupling scheme, we can express the reduced transition dipole moment between the levels $|\beta J\rangle$ and $\left|\beta^{\prime \prime} J^{\prime \prime}\right\rangle$ as a function of the monoelectronic transition dipole moment (MTDM) $\left\langle n_{2}^{\prime \prime} \ell_{2}^{\prime \prime}|\hat{r}| n_{2} \ell_{2}\right\rangle$ expressed as the matrix element of the monoelectronic $\hat{r}$ operator. We apply the following successive steps [49].

By writing atomic levels as the lists of quantum numbers $\left|n_{1} \ell_{1} n_{2} \ell_{2} L_{v} S_{v} J_{v} J_{c} J\right\rangle$ (and similarly for double-primed quantum numbers), we start working with $\left(J_{c}, J_{v}, J\right)$,

$$
\begin{aligned}
\left|\left\langle n_{1} \ell_{1} n_{2}^{\prime \prime} \ell_{2}^{\prime \prime} L_{v}^{\prime \prime} S_{v} J_{v}^{\prime \prime} J_{c} J^{\prime \prime}\|\mathbf{d}\| n_{1} \ell_{1} n_{2} \ell_{2} L_{v} S_{v} J_{v} J_{c} J\right\rangle\right|^{2} \\
=(2 J+1)\left(2 J^{\prime \prime}+1\right)\left\{\begin{array}{ccc}
J_{v} & J_{c} & J \\
J^{\prime \prime} & 1 & J_{v}^{\prime \prime}
\end{array}\right\}^{2} \\
\quad \times\left|\left\langle n_{1} \ell_{1} n_{2}^{\prime \prime} \ell_{2}^{\prime \prime} L_{v}^{\prime \prime} S_{v} J_{v}^{\prime \prime}\|\mathbf{d}\| n_{1} \ell_{1} n_{2} \ell_{2} L_{v} S_{v} J_{v}\right\rangle\right|^{2} .
\end{aligned}
$$

Then we go one step further, with $\left(L_{v}, S_{v}, J_{v}\right)$,

$$
\begin{aligned}
\left|\left\langle n_{1} \ell_{1} n_{2}^{\prime \prime} \ell_{2}^{\prime \prime} L_{v}^{\prime \prime} S_{v} J_{v}^{\prime \prime}\|\mathbf{d}\| n_{1} \ell_{1} n_{2} \ell_{2} L_{v} S_{v} J_{v}\right\rangle\right|^{2} \\
=\left(2 J_{v}+1\right)\left(2 J_{v}^{\prime \prime}+1\right)\left\{\begin{array}{ccc}
L_{v} & S_{v} & J_{v} \\
J_{v}^{\prime \prime} & 1 & L_{v}^{\prime \prime}
\end{array}\right\}^{2} \\
\times\left|\left\langle n_{1} \ell_{1} n_{2}^{\prime \prime} \ell_{2}^{\prime \prime} L_{v}^{\prime \prime}\|\mathbf{d}\| n_{1} \ell_{1} n_{2} \ell_{2} L_{v}\right\rangle\right|^{2},
\end{aligned}
$$

and with $\left(n_{1}, \ell_{1}, n_{2}, \ell_{2}, L_{v}\right)$,

$$
\begin{aligned}
\left|\left\langle n_{1} \ell_{1} n_{2}^{\prime \prime} \ell_{2}^{\prime \prime} L_{v}^{\prime \prime}\|\mathbf{d}\| n_{1} \ell_{1} n_{2} \ell_{2} L_{v}\right\rangle\right|^{2} \\
=\left(1+\delta_{n_{1} n_{2}} \delta_{\ell_{1} \ell_{2}}\right)\left(1+\delta_{n_{1} n_{2}^{\prime \prime}} \delta_{\ell_{1} \ell_{2}^{\prime \prime}}\right)\left(2 L_{v}+1\right) \\
\quad \times\left(2 L_{v}^{\prime \prime}+1\right)\left\{\begin{array}{ccc}
\ell_{2} & \ell_{1} & L_{v} \\
L_{v}^{\prime \prime} & 1 & \ell_{2}^{\prime \prime}
\end{array}\right\}^{2}\left|\left\langle n_{2}^{\prime \prime} \ell_{2}^{\prime \prime}\|\mathbf{d}\| n_{2} \ell_{2}\right\rangle\right|^{2},
\end{aligned}
$$

where the $\delta$ 's are Kronecker symbols, which bring a factor of 2 for equivalent electrons $\left(n_{1} \ell_{1}\right)=\left(n_{2} \ell_{2}\right)$ or $\left(n_{1} \ell_{1}\right)=\left(n_{2}^{\prime \prime} \ell_{2}^{\prime \prime}\right)$.
Finally,

$$
\begin{aligned}
\left|\left\langle n_{2}^{\prime \prime} \ell_{2}^{\prime \prime}\|\mathbf{d}\| n_{2} \ell_{2}\right\rangle\right|^{2}= & e^{2} r_{n_{2} \ell_{2}, n_{2}^{\prime \prime} \ell_{2}^{\prime \prime}}^{2}\left(2 \ell_{2}+1\right) \\
& \times\left(2 \ell_{2}^{\prime \prime}+1\right)\left(\begin{array}{ccc}
\ell_{2}^{\prime \prime} & 1 & \ell_{2} \\
0 & 0 & 0
\end{array}\right)^{2},
\end{aligned}
$$

where (:::) is a Wigner 3-j symbol, $e$ is the absolute value of the electronic charge, and $r_{n_{2} \ell_{2}, n_{2}^{\prime \prime} \ell_{2}^{\prime \prime}}=r_{n_{2}^{\prime \prime} \ell_{2}^{\prime \prime}, n_{2} \ell_{2}} \equiv\left\langle n_{2}^{\prime \prime} \ell_{2}^{\prime \prime}|\hat{r}| n_{2} \ell_{2}\right\rangle$.

\section{Real part of the polarizability}

We assume that the polarizability $\alpha_{k}(\omega)$ of level $|\beta J\rangle$ [see Eq. (4)] involves transitions towards levels $\left|\beta^{\prime \prime} J^{\prime \prime}\right\rangle$ belonging to configurations of the kind $4 f^{q} . n_{1} \ell_{1} . n_{2}^{\prime \prime} \ell_{2}^{\prime \prime}$. By separating the contributions of those configurations, we can write

$$
\alpha_{k}(\omega)=\sum_{n_{2}^{\prime \prime} \ell_{2}^{\prime \prime}} \alpha_{k}^{n_{2}^{\prime \prime} \ell_{2}^{\prime \prime}}(\omega),
$$

which relies on two main hypotheses: (i) Transitions to levels of configurations in which one core electron is excited, e.g., $4 f^{q-1} .5 d . n_{1} \ell_{1} . n_{2} \ell_{2}$, are excluded, as they are often significantly weaker. (ii) Configuration interaction is totally neglected, both between different configurations of the kind $4 f^{q} \cdot n_{1} \ell_{1} \cdot n_{2}^{\prime \prime} \ell_{2}^{\prime \prime}$ and with those of the kind $4 f^{q-1} . n^{\prime \prime} \ell^{\prime \prime} . n_{1} \ell_{1} . n_{2} \ell_{2}$. The effect of CI is addressed in the next subsection.

The central assumption of this work is that the energy differences implying the levels of a given configuration can be replaced with a single effective energy $\hbar \omega_{n_{2}^{\prime \prime} \ell_{2}^{\prime \prime}}$ :

$$
E_{\beta^{\prime \prime} J^{\prime \prime}}-E_{\beta J} \approx \hbar \omega_{n_{2}^{\prime \prime} \ell_{2}^{\prime \prime}} \text {. }
$$

The validity of this assumption depends on the frequency $\omega$ at which the DDPs are calculated, which should not "fall" into the levels of the $4 f^{q} . n_{1} \ell_{1} . n_{2}^{\prime \prime} \ell_{2}^{\prime \prime}$ configuration. If we denote $\min \left(E_{\beta^{\prime \prime} J^{\prime \prime}}\right)$ and $\max \left(E_{\beta^{\prime \prime} J^{\prime \prime}}\right)$ their lowest and highest energies, Eq. (18) is not applicable for

$$
\min \left(E_{\beta^{\prime \prime} J^{\prime \prime}}\right)-E_{\beta J} \lesssim \bar{\omega} \lesssim \max \left(E_{\beta^{\prime \prime} J^{\prime \prime}}\right)-E_{\beta J},
$$

where $\bar{\omega}= \pm \omega$ for $E_{\beta^{\prime \prime} J^{\prime \prime}}>E_{\beta J}$ and $E_{\beta^{\prime \prime} J^{\prime \prime}}<E_{\beta J}$, respectively. For ground-level Ho, the excluded frequencies, which correspond to the energies of the $4 f^{11}\left({ }^{4} I_{15 / 2}^{o}\right) \cdot 6 s 6 p\left({ }^{1} P_{1}^{o}\right)(15 / 2,1)$ manifold, roughly range from 23000 to $24000 \mathrm{~cm}^{-1}$.

Consequently, the sum in Eq. (4) is restricted to the quantum numbers $L_{v}^{\prime \prime},{ }^{\prime} J_{v}^{\prime \prime}$, and $J^{\prime \prime}$ allowed by electric-dipole transitions. (For configurations with at least one $s$ electron, there is obviously only one possible $L_{v}$ value.) Inserting Eq. (17) into Eq. (5), we can extract the real part:

$$
\begin{aligned}
\operatorname{Re}\left[\alpha_{k}^{n_{2}^{\prime \prime} \ell_{2}^{\prime \prime}}(\omega)\right]= & \frac{2\left(\omega_{n_{2}^{\prime \prime} \ell_{2}^{\prime \prime}} \delta_{(-1)^{k}, 1}-\omega \delta_{(-1)^{k},-1}\right)}{\hbar\left(\omega_{n_{2}^{\prime \prime} \ell_{2}^{\prime \prime}}^{2}-\omega^{2}\right)} \sqrt{2 k+1} \sum_{L_{v}^{\prime \prime} J_{v}^{\prime \prime} J^{\prime \prime}}(-1)^{J+J^{\prime \prime}}\left\{\begin{array}{ccc}
1 & 1 & k \\
J & J & J^{\prime \prime}
\end{array}\right\} \\
& \times\left|\left\langle n_{1} \ell_{1} n_{2}^{\prime \prime} \ell_{2}^{\prime \prime} L_{v}^{\prime \prime} S_{v} J_{v}^{\prime \prime} J_{c} J^{\prime \prime}\|\mathbf{d}\| n_{1} \ell_{1} n_{2} \ell_{2} L_{v} S_{v} J_{v} J_{c} J\right\rangle\right|^{2} .
\end{aligned}
$$

Using Eq. (13), we obtain

$$
\begin{aligned}
\operatorname{Re}\left[\alpha_{k}^{n_{2}^{\prime \prime} \ell_{2}^{\prime \prime}}(\omega)\right]= & \frac{2\left(\omega_{n_{2}^{\prime \prime} \ell_{2}^{\prime \prime}} \delta_{(-1)^{k}, 1}-\omega \delta_{(-1)^{k},-1}\right)}{\hbar\left(\omega_{n_{2}^{\prime \prime} \ell_{2}^{\prime \prime}}^{2}-\omega^{2}\right)} \sqrt{2 k+1} \sum_{L_{v}^{\prime \prime} J_{v}^{\prime \prime} J^{\prime \prime}}(-1)^{J+J^{\prime \prime}}\left\{\begin{array}{ccc}
1 & 1 & k \\
J & J & J^{\prime \prime}
\end{array}\right\} \\
& \times(2 J+1)\left(2 J^{\prime \prime}+1\right)\left\{\begin{array}{ccc}
J_{v} & J_{c} & J \\
J^{\prime \prime} & 1 & J_{v}^{\prime \prime}
\end{array}\right\}^{2}\left|\left\langle n_{1} \ell_{1} n_{2}^{\prime \prime} \ell_{2}^{\prime \prime} L_{v}^{\prime \prime} S_{v} J_{v}^{\prime \prime}\|\mathbf{d}\| n_{1} \ell_{1} n_{2} \ell_{2} L_{v} S_{v} J_{v}\right\rangle\right|^{2} .
\end{aligned}
$$


To calculate this expression, we note that the quantum number $J^{\prime \prime}$ only appears in angular terms, so that we use the identity (see Ref. [52], p. 305)

$$
\sum_{X}(-1)^{R+X}(2 X+1)\left\{\begin{array}{lll}
a & b & p \\
c & d & X
\end{array}\right\}\left\{\begin{array}{lll}
c & d & q \\
e & f & X
\end{array}\right\}\left\{\begin{array}{lll}
e & f & r \\
b & a & X
\end{array}\right\}=\left\{\begin{array}{lll}
p & q & r \\
e & a & d
\end{array}\right\}\left\{\begin{array}{lll}
p & q & r \\
f & b & c
\end{array}\right\},
$$

with $R=a+b+c+d+e+f+p+q+r$, as well as the invariance properties of Wigner 6-j symbols with respect to line and column permutations. Applying Eq. (22) with $a=d=1, b=c=J, e=J_{v}^{\prime \prime}, f=J_{c}, p=k$, and $q=r=J_{v}$, we can get rid of $J^{\prime \prime}$ in Eq. (21):

$$
\begin{aligned}
\operatorname{Re}\left[\alpha_{k}^{n_{2}^{\prime \prime} \ell_{2}^{\prime \prime}}(\omega)\right]= & \frac{2\left(\omega_{n_{2}^{\prime \prime} \ell_{2}^{\prime \prime}} \delta_{(-1)^{k}, 1}-\omega \delta_{(-1)^{k},-1}\right)}{\hbar\left(\omega_{n_{2}^{\prime \prime} \ell_{2}^{\prime \prime}}^{2}-\omega^{2}\right)} \sqrt{2 k+1} \sum_{L_{v}^{\prime \prime} J_{v}^{\prime \prime}}(-1)^{J_{c}+2 J_{v}+J_{v}^{\prime \prime}+J+k} \\
& \times(2 J+1)\left\{\begin{array}{ccc}
1 & 1 & k \\
J_{v} & J_{v} & J_{v}^{\prime \prime}
\end{array}\right\}\left\{\begin{array}{ccc}
J_{v} & J_{c} & J \\
J & k & J_{v}
\end{array}\right\}\left|\left\langle n_{1} \ell_{1} n_{2}^{\prime \prime} \ell_{2}^{\prime \prime} L_{v}^{\prime \prime} S_{v} J_{v}^{\prime \prime}\|\mathbf{d}\| n_{1} \ell_{1} n_{2} \ell_{2} L_{v} S_{v} J_{v}\right\rangle\right|^{2} .
\end{aligned}
$$

At this point, it is the following fact is noteworthy [53]. The definitions of the coupled polarizabilities $\alpha_{k}(\omega)$ and $\alpha_{k}^{n_{2}^{\prime \prime} \ell_{2}^{\prime \prime}}(\omega)$, given, respectively, by Eqs. (4) and (17), are such that they can be written as the reduced matrix elements of the operators $\hat{\alpha}_{k}(\omega)$ and $\hat{\alpha}_{k}^{n_{2}^{\prime \prime} \ell_{2}^{\prime \prime}}(\omega)$, which are tensors of rank $k$. In particular, one can resort to the Wigner-Eckart theorem [52] to calculate the coupled polarizability of a level $|\beta J M\rangle$, namely, $\left\langle\beta J M\left|\hat{\alpha}_{k}(\omega)\right| \beta J M\right\rangle=\left\langle\beta J\left\|\hat{\alpha}_{k}(\omega)\right\| \beta J\right\rangle \times C_{J M k 0}^{J M} / \sqrt{2 J+1}$, with $C_{J M k 0}^{J M}$ a Clebsh-Gordan coefficient [and similarly for $\hat{\alpha}_{k}^{n_{2}^{\prime \prime} \ell_{2}^{\prime \prime}}(\omega)$ ]. One can also apply the transformation of tensor operators regarding angular-momentum basis sets; in this respect, Eq. (23) can be seen as such a transformation,

$$
\begin{aligned}
& \left\langle n_{1} \ell_{1} n_{2} \ell_{2} L_{v} S_{v} J_{v} J_{c} J\left\|\operatorname{Re}\left[\hat{\alpha}_{k}^{n_{2}^{\prime \prime} \ell_{2}^{\prime \prime}}(\omega)\right]\right\| n_{1} \ell_{1} n_{2} \ell_{2} L_{v} S_{v} J_{v} J_{c} J\right\rangle \\
& =(-1)^{J_{c}+J_{v}+k+J}(2 J+1)\left\{\begin{array}{ccc}
J_{v} & J_{c} & J \\
J & k & J_{v}
\end{array}\right\}\left\langle n_{1} \ell_{1} n_{2} \ell_{2} L_{v} S_{v} J_{v}\left\|\operatorname{Re}\left[\hat{\alpha}_{k}^{n_{2}^{\prime \prime} \ell_{2}^{\prime \prime}}(\omega)\right]\right\| n_{1} \ell_{1} n_{2} \ell_{2} L_{v} S_{v} J_{v}\right\rangle,
\end{aligned}
$$

where

$$
\begin{aligned}
& \left\langle n_{1} \ell_{1} n_{2} \ell_{2} L_{v} S_{v} J_{v}\left\|\operatorname{Re}\left[\hat{\alpha}_{k}^{n_{2}^{\prime \prime} \ell_{2}^{\prime \prime}}(\omega)\right]\right\| n_{1} \ell_{1} n_{2} \ell_{2} L_{v} S_{v} J_{v}\right\rangle \\
& \quad=\frac{2\left(\omega_{n_{2}^{\prime \prime} \ell_{2}^{\prime \prime}} \delta_{(-1)^{k}, 1}-\omega \delta_{\left.(-1)^{k},-1\right)}\right)}{\hbar\left(\omega_{n_{2}^{\prime \prime} \ell_{2}^{\prime \prime}}^{2}-\omega^{2}\right)} \sqrt{2 k+1} \sum_{J_{v}^{\prime \prime} L_{v}^{\prime \prime}}(-1)^{J_{v}+J_{v}^{\prime \prime}}\left\{\begin{array}{ccc}
1 & 1 & k \\
J_{v} & J_{v} & J_{v}^{\prime \prime}
\end{array}\right\}\left|\left\langle n_{1} \ell_{1} n_{2}^{\prime \prime} \ell_{2}^{\prime \prime} L_{v}^{\prime \prime} S_{v} J_{v}^{\prime \prime}\|\mathbf{d}\| n_{1} \ell_{1} n_{2} \ell_{2} L_{v} S_{v} J_{v}\right\rangle\right|^{2} .
\end{aligned}
$$

Returning to our main purpose, we apply Eq. (22) twice more: first, with Eq. (14) to express the sum over $J_{v}^{\prime \prime}$ and, second, with Eq. (15) to express the sum over $L_{v}^{\prime \prime}$. Doing so, we get to the final expression,

$$
\begin{aligned}
\operatorname{Re}\left[\alpha_{k}^{n_{2}^{\prime \prime} \ell_{2}^{\prime \prime}}(\omega)\right]= & \frac{2 \sqrt{2 k+1}}{\hbar} \times \frac{\omega_{n_{2}^{\prime \prime} \ell_{2}^{\prime \prime}} \delta_{(-1)^{k}, 1}-\omega \delta_{(-1)^{k},-1}}{\omega_{n_{2}^{\prime \prime} \ell_{2}^{\prime \prime}}^{2}-\omega^{2}}\left(1+\delta_{n_{1} n_{2}} \delta_{\ell_{1} \ell_{2}}\right) \\
& \times\left(1+\delta_{n_{1} n_{2}^{\prime \prime}} \delta_{\ell_{1} \ell_{2}^{\prime \prime}}\right)(-1)^{J+J_{c}-S_{v}+\ell_{1}+\ell_{2}^{\prime \prime}+k}(2 J+1)\left(2 J_{v}+1\right)\left(2 L_{v}+1\right)\left(2 \ell_{2}+1\right)\left(2 \ell_{2}^{\prime \prime}+1\right) \\
& \times\left\{\begin{array}{ccc}
J_{v} & J_{c} & J \\
J & k & J_{v}
\end{array}\right\}\left\{\begin{array}{ccc}
L_{v} & S_{v} & J_{v} \\
J_{v} & k & L_{v}
\end{array}\right\}\left\{\begin{array}{ccc}
\ell_{2} & \ell_{1} & L_{v} \\
L_{v} & k & \ell_{2}
\end{array}\right\}\left\{\begin{array}{ccc}
1 & 1 & k \\
\ell_{2} & \ell_{2} & \ell_{2}^{\prime \prime}
\end{array}\right\}\left(\begin{array}{ccc}
\ell_{2}^{\prime \prime} & 1 & \ell_{2} \\
0 & 0 & 0
\end{array}\right)^{2} e^{2} r_{n_{2} \ell_{2}, n_{2}^{\prime \prime} \ell_{2}^{\prime \prime},}
\end{aligned}
$$

which depends on two effective parameters: the transition

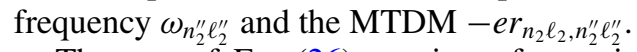

The rest of Eq. (26) consists of very insightful angular terms. In particular, the 6- $j$ symbols indicate that, if one of the quantum numbers $J, J_{v}$, or $L_{v}$ is equal to 0 , then the vector and tensor polarizabilities, proportional to $\operatorname{Re}\left[\alpha_{k=1}^{n_{2}^{\prime \prime} \ell_{2}^{\prime \prime}}(\omega)\right]$ and $\left.\operatorname{Re}\left[\alpha_{k=2}^{n_{2}^{\prime \prime} \ell_{2}^{\prime \prime}}(\omega)\right]\right)$, respectively, vanish. This is, for instance, the case for lanthanides at their ground level, which is characterized by $L_{v}=J_{v}=0$. In our full numerical calculation of the polarizability $[45,46]$, we have shown that indeed the vector and tensor contributions are much weaker than the scalar one. Equation (26) tends to confirm that those weak contributions come from transitions in which one $4 f$ electron is excited.
Such conclusions are also valid for any level belonging to the lowest configuration $4 f^{q} 6 s^{2}$, as shown in our previous articles (see Ref. [46] and Sec. III C here).

\section{Imaginary part of the polarizability}

For the imaginary part to be relevant, we consider a metastable level $|\beta J\rangle$, i.e., whose natural line width $\gamma_{\beta J}$ is negligible compared to the photon-scattering rate induced by the electromagnetic field $[44,45]$. In practice, this may concern excited levels of the lowest configuration $4 f^{q} 6 s^{2}$ or the levels $4 f^{q}\left({ }^{2 S_{c}+1} L_{c J_{c}}\right) \cdot 6 s 6 p\left({ }^{3} P_{2}\right)\left(J_{c}, 2\right)_{J_{c}+2}$, which have no decay channel in the E1 approximation [except for level $(6,2)_{8}^{o}$ of Er] [54]. 
As Eq. (6) shows, the imaginary part of the polarizability involves the natural line width of intermediate levels $\left|\beta^{\prime \prime} J^{\prime \prime}\right\rangle$,

$$
\begin{aligned}
\gamma_{\beta^{\prime \prime} J^{\prime \prime}} & =\sum_{\tilde{\beta} \tilde{J}, E_{\tilde{\beta} \tilde{J}}<E_{\beta^{\prime \prime} J^{\prime \prime}}} A_{\beta^{\prime \prime} J^{\prime \prime}, \tilde{\beta} \tilde{J}} \\
& =\frac{\sum_{\tilde{\beta} \tilde{J}}\left(E_{\beta^{\prime \prime} J^{\prime \prime}}-E_{\tilde{\beta} \tilde{J}}\right)^{3}\left|\left\langle\beta^{\prime \prime} J^{\prime \prime}\|\mathbf{d}\| \tilde{\beta} \tilde{J}\right\rangle\right|^{2}}{3 \pi \epsilon_{0} \hbar^{4} c^{3}\left(2 J^{\prime \prime}+1\right)},
\end{aligned}
$$

where $A_{\beta^{\prime \prime} J^{\prime \prime}, \tilde{\beta} \tilde{J}}$ is the transition probability characterizing the spontaneous emission from level $\left|\beta^{\prime \prime} J^{\prime \prime}\right\rangle$ to level $|\tilde{\beta} \tilde{J}\rangle$. We focus on the influence of the $\beta^{\prime \prime} J^{\prime \prime}$ levels belonging to the configuration $4 f^{q} . n_{1} \ell_{1} . n_{2}^{\prime \prime} \ell_{2}^{\prime \prime}$. In addition, we assume that the latter levels only decay towards levels $|\tilde{\beta} \tilde{J}\rangle$ belonging to the configuration $4 f^{q} \cdot n_{1} \ell_{1} \cdot n_{2} \ell_{2}$. Therefore the sum in Eq. (27) runs over the quantum numbers $\tilde{J}, \tilde{J}_{v}$, and $\tilde{L}_{v}$. If we express the squared reduced transition dipole moment as in Eq. (13), Eq. (27) becomes

$$
\begin{aligned}
\gamma_{L_{v}^{\prime \prime} J_{v}^{\prime \prime} J^{\prime \prime}}= & \frac{\omega_{n_{2}^{\prime \prime} \ell_{2}^{\prime \prime}}^{3}}{3 \pi \epsilon_{0} \hbar c^{3}} \sum_{\tilde{L}_{v} \tilde{J}_{v} \tilde{J}}(2 \tilde{J}+1)\left\{\begin{array}{ccc}
\tilde{J}_{v} & J_{c} & \tilde{J} \\
J^{\prime \prime} & 1 & J_{v}^{\prime \prime}
\end{array}\right\}^{2} \\
& \times\left|\left\langle n_{1} \ell_{1} n_{2}^{\prime \prime} \ell_{2}^{\prime \prime} L_{v}^{\prime \prime} S_{v} J_{v}^{\prime \prime}\|\mathbf{d}\| n_{1} \ell_{1} n_{2} \ell_{2} \tilde{L}_{v} S_{v} \tilde{J}_{v}\right\rangle\right|^{2} .
\end{aligned}
$$

Since $\tilde{J}$ only appears in angular factors, the sum over $\tilde{J}$ reduces to the orthogonalization relations of $6-j$ symbols,

$$
\sum_{\tilde{J}}(2 \tilde{J}+1)\left\{\begin{array}{ccc}
\tilde{J}_{v} & J_{c} & \tilde{J} \\
J^{\prime \prime} & 1 & J_{v}^{\prime \prime}
\end{array}\right\}^{2}=\frac{1}{2 J_{v}^{\prime \prime}+1} .
$$

Using Eqs. (14) and (15) for the transition dipole moment, we can calculate the sums over $\tilde{J}_{v}$ and $\tilde{L}_{v}$ in a similar way, and finally, we get to the expression [see also Eq. (16)]

$$
\begin{aligned}
\gamma_{L_{v}^{\prime \prime} J_{v}^{\prime \prime} J^{\prime \prime}}= & \frac{\omega_{n_{2}^{\prime \prime} \ell_{2}^{\prime \prime}}^{3} e^{2} r_{n_{2} \ell_{2}, n_{2}^{\prime \prime} \ell_{2}^{\prime \prime}}^{2}}{3 \pi \epsilon_{0} \hbar c^{3}}\left(2 \ell_{2}+1\right)\left(\begin{array}{ccc}
\ell_{2}^{\prime \prime} & 1 & \ell_{2} \\
0 & 0 & 0
\end{array}\right)^{2} \\
& \times\left(1+\delta_{n_{1} n_{2}} \delta_{\ell_{1} \ell_{2}}\right)\left(1+\delta_{n_{1} n_{2}^{\prime \prime}} \delta_{\ell_{1} \ell_{2}^{\prime \prime}}\right)
\end{aligned}
$$

Strikingly, the natural line width of the intermediate levels does not depend on $L_{v}^{\prime \prime}, J_{v}^{\prime \prime}$, or $J^{\prime \prime}$; it is identical for all the levels of the $4 f^{q} . n_{1} \ell_{1} . n_{2}^{\prime \prime} \ell_{2}^{\prime \prime}$ configuration. In calculating $\operatorname{Im}\left[\alpha_{k}^{n_{2}^{\prime \prime} \ell_{2}^{\prime \prime}}(\omega)\right]$, we can factorize $\gamma_{L_{v}^{\prime \prime} J_{v}^{\prime \prime} J^{\prime \prime}}$ out of the sum over $L_{v}^{\prime \prime}, J_{v}^{\prime \prime}$, and $J^{\prime \prime}$, and so steps similar [see Eqs. (21)-(26)] to those for the real part can be applied, which leads to the final expression

$$
\begin{aligned}
\operatorname{Im}\left[\alpha_{k}^{n_{2}^{\prime \prime} \ell_{2}^{\prime \prime}}(\omega)\right]= & \frac{\omega_{n_{2}^{\prime \prime} \ell_{2}^{\prime \prime}}^{3} \sqrt{2 k+1}}{3 \pi \epsilon_{0} \hbar^{2} c^{3}} \times \frac{\left(\omega_{n_{2}^{\prime \prime} \ell_{2}^{\prime \prime}}^{2}+\omega^{2}\right) \delta_{(-1)^{k}, 1}-2 \omega \omega_{n_{2}^{\prime \prime} \ell_{2}^{\prime \prime}} \delta_{(-1)^{k},-1}}{\left(\omega_{n_{2}^{\prime \prime} \ell_{2}^{\prime \prime}}^{2}-\omega^{2}\right)^{2}}\left(1+\delta_{n_{1} n_{2}} \delta_{\ell_{1} \ell_{2}}\right)^{2}\left(1+\delta_{n_{1} n_{2}^{\prime \prime}} \delta_{\ell_{1} \ell_{2}^{\prime \prime}}\right)^{2} \\
& \times(-1)^{J+J_{c}-S_{v}+\ell_{1}+\ell_{2}^{\prime \prime}+k}(2 J+1)\left(2 J_{v}+1\right)\left(2 L_{v}+1\right)\left(2 \ell_{2}+1\right)^{2}\left(2 \ell_{2}^{\prime \prime}+1\right) \\
& \times\left\{\begin{array}{ccc}
J_{v} & J_{c} & J \\
J & k & J_{v}
\end{array}\right\}\left\{\begin{array}{ccc}
L_{v} & S_{v} & J_{v} \\
J_{v} & k & L_{v}
\end{array}\right\}\left\{\begin{array}{ccc}
\ell_{2} & \ell_{1} & L_{v} \\
L_{v} & k & \ell_{2}
\end{array}\right\}\left\{\begin{array}{ccc}
1 & 1 & k \\
\ell_{2} & \ell_{2} & \ell_{2}^{\prime \prime}
\end{array}\right\}\left(\begin{array}{ccc}
\ell_{2}^{\prime \prime} & 1 & \ell_{2} \\
0 & 0 & 0
\end{array}\right)^{4} e^{4} r_{n_{2} \ell_{2}, n_{2}^{\prime \prime} \ell_{2}^{\prime \prime} \cdot}
\end{aligned}
$$

Therefore, similarly to the real part, the imaginary part of the polarizability depends on the effective frequency $\omega_{n_{2}^{\prime \prime} \ell_{2}^{\prime \prime}}$ and the monoelectronic transition dipole moment $-e r_{n_{2}} \ell_{2}, n_{2}^{\prime \prime} \ell_{2}^{\prime \prime}$ between the two configurations and on some angular factors.

Again, those angular factors show that, if one of the quantum numbers $J, J_{v}$, or $L_{v}$ is equal to 0 , then the vector and tensor polarizabilities are equal to 0 . For lanthanide atoms in the ground level, our simplified model predicts that both the real and the imaginary parts of the vector and tensor DDPs vanish [see, respectively, Eqs. (26) and (31)]. For the real part, that prediction agrees with our full numerical calculation $[45,46]$ (see also Sec. III C), but for the imaginary part it does not. To explain this contradiction, we note that the vector-to-scalar and tensor-to-scalar ratios are significantly higher for Er than for Dy and Ho. In addition, Er is the only atom among the three for which we modeled the excited levels including the configurations $4 f^{11} 5 d 6 s^{2}, 4 f^{11} 5 d^{2} 6 s$, and $4 f^{12} 6 s 6 p$, and so we expect to have a better description of CI mixing for it. This tends to prove that CI plays an important role in the imaginary part of the DDPs. That is why, in the next subsection, we improve our model by taking into account CIs among excited levels.

\section{Effect of configuration interaction}

We focus on the polarizability of the ground level of lanthanides, denoted $|\beta J\rangle \equiv|0 J\rangle$ and characterized by
$L_{v}=S_{v}=0$ and $J=J_{c}$. According to Eqs. (26) and (31), there are three excited levels, denoted $\left|1 J^{\prime \prime}\right\rangle$ for $J^{\prime \prime}=J$ and $J \pm 1$, which contribute to the polarizability; they are characterized by $L_{v}^{\prime \prime}=J_{v}^{\prime \prime}=1$. In this section, we consider that these levels can be mixed by CI to other levels $\left|m J^{\prime \prime}\right\rangle$ belonging to other configurations. Therefore the eigenvector of the excited levels $\left|\beta^{\prime \prime} J^{\prime \prime}\right\rangle$ can be expanded as

$$
\left|\beta^{\prime \prime} J^{\prime \prime}\right\rangle=\sum_{m \geqslant 1} c_{\beta^{\prime \prime} m}^{\left(J^{\prime \prime}\right)}\left|m J^{\prime \prime}\right\rangle
$$

where $\left|m J^{\prime \prime}\right\rangle$ are henceforth called basis states. Furthermore, we assume that state $\left|1 J^{\prime \prime}\right\rangle$ is the only one contributing to the transition dipole moment $\left\langle 0 J\|\mathbf{d}\| \beta^{\prime \prime} J^{\prime \prime}\right\rangle$. This is exactly valid for basis states of the $4 f^{q-1} 5 d^{2} 6 s$ configuration and approximately valid for states of the $4 f^{q-1} 5 d 6 s^{2}$ configuration, as the latter contribute significantly less than states of the $4 f^{q} 6 s 6 p$ configuration.

In this case the squared transition dipole moment reads

$$
\begin{aligned}
\left|\left\langle\beta^{\prime \prime} J^{\prime \prime}\|\mathbf{d}\| 0 J\right\rangle\right|^{2} & =\left|c_{\beta^{\prime \prime}, 1}^{\left(J^{\prime \prime}\right)}\right|^{2}\left|\left\langle 1 J^{\prime \prime}\|\mathbf{d}\| 0 J\right\rangle\right|^{2} \\
& =\frac{2}{3} w_{\beta^{\prime \prime}, 1}^{\left(J^{\prime \prime}\right)}\left(2 J^{\prime \prime}+1\right) r_{6 s 6 p}^{2},
\end{aligned}
$$

where, in the second line, we have expressed $\left|\left\langle 1 J^{\prime \prime}\|\mathbf{d}\| 0 J\right\rangle\right|^{2}$ using Eqs. (13)-(16) and the explicit forms of the $3-j$ and 
6-j symbols. In Eq. (33) we introduced the weights $w_{\beta^{\prime \prime}, 1}^{\left(J^{\prime \prime}\right)}=$ $\left|c_{\beta^{\prime \prime}, 1}^{\left(J^{\prime \prime}\right)}\right|^{2}$ of the basis states $\left|1 J^{\prime \prime}\right\rangle$ in levels $\left|\beta^{\prime \prime} J^{\prime \prime}\right\rangle$, which satisfy the normalization conditions

$$
\sum_{m \geqslant 1} w_{\beta^{\prime \prime} m}^{\left(J^{\prime \prime}\right)}=\sum_{\beta^{\prime \prime}} w_{\beta^{\prime \prime} m}^{\left(J^{\prime \prime}\right)}=1
$$

for each $J^{\prime \prime}$ separately.

Turning to the polarizability, we find that the real part is

$$
\begin{aligned}
\operatorname{Re} & {\left[\alpha_{k}^{6 p}(\omega)\right] } \\
& =\frac{4 e^{2} r_{6 s 6 p}^{2} \sqrt{2 k+1}}{3 \hbar} \frac{\omega_{6 p} \delta_{(-1)^{k}, 1}-\omega \delta_{(-1)^{k},-1}}{\omega_{6 p}^{2}-\omega^{2}} \\
& \times \sum_{J^{\prime \prime}}(-1)^{J+J^{\prime \prime}}\left(2 J^{\prime \prime}+1\right)\left\{\begin{array}{ccc}
1 & 1 & k \\
J & J & J^{\prime \prime}
\end{array}\right\} \sum_{\beta^{\prime \prime}} w_{\beta^{\prime \prime}, 1}^{\left(J^{\prime \prime}\right)} \\
& =-\frac{4 e^{2} r_{6 s 6 p}^{2} \omega_{6 p} \delta_{k 0}}{\hbar\left(\omega_{6 p}^{2}-\omega^{2}\right)} \sqrt{\frac{2 J+1}{3}},
\end{aligned}
$$

where the sums over $\beta^{\prime \prime}$ and $J^{\prime \prime}$ are calculated using, respectively, Eq. (34) and (see Ref. [52], p. 305)

$$
\begin{aligned}
& \sum_{X}(-1)^{a+b+X}(2 X+1)\left\{\begin{array}{lll}
a & a & c \\
b & b & X
\end{array}\right\} \\
& =\delta_{c 0} \sqrt{(2 a+1)(2 b+1)},
\end{aligned}
$$

with $a=1, b=J, c=k$, and $X=J^{\prime \prime}$. Equation (35) shows that the vector and tensor polarizabilities vanish for lanthanides in the ground level (or in any level of the electronic configuration $4 f^{q} 6 s^{2}$ ), whatever the CI mixing in the excited levels; the only contribution is thus the scalar one, $\operatorname{Re}\left[\alpha_{\text {scal }}(\omega)\right]=$ $4 e^{2} r_{6 s 6 p}^{2} \omega_{6 p} / 3 \hbar\left(\omega_{6 p}^{2}-\omega^{2}\right)$. In this respect the inclusion of CI in our model does not modify the conclusions in the singleconfiguration case [see Eq. (26)]. This confirms that, as shown in our full numerical calculations in Sec. III, the vector and tensor contributions arise from the $4 f-5 d$ transitions between the configurations $4 f^{q} 6 s^{2}$ and $4 f^{q-1} 5 d 6 s^{2}$, depending on $r_{4 f 5 d}$. By contrast, in Eq. (35), even if levels $\left|\beta^{\prime \prime} J^{\prime \prime}\right\rangle$ may contain some $4 f^{q-1} 5 d 6 s^{2}$ character, the contribution of the $4 f^{q} 6 s^{2}-4 f^{q} 6 s 6 p$ transition is the only one considered in $\left|\left\langle\beta^{\prime \prime} J^{\prime \prime}\|\mathbf{d}\| 0 J\right\rangle\right|^{2}$ [see Eq. (33)].

In order to calculate the imaginary part of the polarizability, we recall that the excited level $\left|1 J^{\prime \prime}\right\rangle$ can only decay toward the ground level $|0 J\rangle$. Therefore $\operatorname{Im}\left[\alpha_{k}^{6 p}(\omega)\right]$ reads

$$
\begin{aligned}
\operatorname{Im}\left[\alpha_{k}^{6 p}(\omega)\right] & =\frac{\left(\omega_{6 p}^{2}+\omega^{2}\right) \delta_{(-1)^{k}, 1}-2 \omega \omega_{6 p} \delta_{(-1)^{k},-1}}{\left(\omega_{6 p}^{2}-\omega^{2}\right)^{2}} \\
& \times \frac{\omega_{6 p}^{3} \sqrt{2 k+1}}{3 \pi \epsilon_{0} \hbar^{2} c^{3}} \sum_{J^{\prime \prime}}(-1)^{J+J^{\prime \prime}}\left\{\begin{array}{ccc}
1 & 1 & k \\
J & J & J^{\prime \prime}
\end{array}\right\} \\
& \times \sum_{\beta^{\prime \prime}} \frac{1}{2 J^{\prime \prime}+1}\left|\left\langle\beta^{\prime \prime} J^{\prime \prime}\|\mathbf{d}\| 0 J\right\rangle\right|^{4} \\
& =\frac{\left(\omega_{6 p}^{2}+\omega^{2}\right) \delta_{(-1)^{k}, 1}-2 \omega \omega_{6 p} \delta_{(-1)^{k},-1}}{\left(\omega_{6 p}^{2}-\omega^{2}\right)^{2}}
\end{aligned}
$$

$$
\begin{aligned}
& \times \frac{4 \omega_{6 p}^{3} r_{6 s 6 p}^{4} \sqrt{2 k+1}}{27 \pi \epsilon_{0} \hbar^{2} c^{3}} \sum_{J^{\prime \prime}}(-1)^{J+J^{\prime \prime}}\left(2 J^{\prime \prime}+1\right) \\
& \times\left\{\begin{array}{ccc}
1 & 1 & k \\
J & J & J^{\prime \prime}
\end{array}\right\} \sum_{\beta^{\prime \prime}}\left(w_{\beta^{\prime \prime}, 1}^{\left(J^{\prime \prime}\right)}\right)^{2},
\end{aligned}
$$

where we have taken the square of Eq. (33).

Equation (37) is a key result of this work. Contrary to the real part given by Eq. (35), the sum over $\beta^{\prime \prime}$ cannot be simplified in the imaginary part of the polarizability, as it involves the squared weights of the $\left|1 J^{\prime \prime}\right\rangle$ basis vectors in the excited levels $\left|\beta^{\prime \prime} J^{\prime \prime}\right\rangle$. In this respect, we can say that the imaginary part of the polarizability is more sensitive to the details of the atomic structure than the real part.

In particular, taking the square of Eq. (34), we find that

$$
\begin{aligned}
\sum_{\beta^{\prime \prime}}\left(w_{\beta^{\prime \prime} m}^{\left(J^{\prime \prime}\right)}\right)^{2} & =\left(\sum_{\beta^{\prime \prime}} w_{\beta^{\prime \prime} m}^{\left(J^{\prime \prime}\right)}\right)^{2}-2 \sum_{\substack{\beta_{1}^{\prime \prime} \beta_{2}^{\prime \prime} \\
\beta_{1}^{\prime \prime}<\beta_{2}^{\prime \prime}}} w_{\beta_{1}^{\prime \prime} m}^{\left(J^{\prime \prime}\right)} w_{\beta_{2}^{\prime \prime} m}^{\left(J^{\prime \prime}\right)} \\
& =1-2 \sum_{\substack{\beta_{1}^{\prime \prime} \beta_{2}^{\prime \prime} \\
\beta_{1}^{\prime \prime}<\beta_{2}^{\prime \prime}}} w_{\beta_{1}^{\prime \prime} m}^{\left(J^{\prime \prime}\right)} w_{\beta_{2}^{\prime \prime} m}^{\left(J^{\prime \prime}\right)} \leqslant 1,
\end{aligned}
$$

where $\beta_{1}^{\prime \prime}<\beta_{2}^{\prime \prime}$ means $E_{\beta_{1}^{\prime \prime} J^{\prime \prime}}<E_{\beta_{2}^{\prime \prime} J^{\prime \prime}}$, to avoid doublecounting. The inequality comes from the fact that $w_{\beta^{\prime \prime} m}^{\left(J^{\prime \prime}\right)} \geqslant$ $0, \forall m, \beta^{\prime \prime}, J^{\prime \prime}$. The limit for which Eq. (38) is unity corresponds to the case where one weight is unity and all the others are 0 , i.e., there is no CI. In this particular case, the sums over $\beta^{\prime \prime}$ and $J^{\prime \prime}$ in Eq. (37) can be simplified,

$$
\operatorname{Im}\left[\alpha_{k}^{6 p}(\omega)\right]=-\frac{4 \omega_{6 p}^{3}\left(\omega_{6 p}^{2}+\omega^{2}\right) e^{4} r_{6 s 6 p}^{4} \delta_{k 0}}{9 \pi \epsilon_{0} \hbar^{2} c^{3}\left(\omega_{6 p}^{2}-\omega^{2}\right)^{2}} \sqrt{\frac{2 J+1}{3}},
$$

and $\quad$ so $\left.\quad \operatorname{Im} \alpha_{\text {scal }}^{6 p}(\omega)\right]=4 \omega_{6 p}^{3}\left(\omega_{6 p}^{2}+\omega^{2}\right) e^{4} r_{6 s 6 p}^{4} / 27 \pi \epsilon_{0} \hbar^{2} c^{3}$ $\left(\omega_{6 p}^{2}-\omega^{2}\right)^{2}$, which can also be obtained from Eq. (31). By comparing Eqs. (37)-(39), we find that CI has two effects:

(i) It tends to reduce the scalar contribution $\operatorname{Im}\left[\alpha_{0}^{6 p}(\omega)\right]$. Indeed in the limit of strong CI mixing, when $N$ basis states $\left|m J^{\prime \prime}\right\rangle(m=1$ to $N)$ are equally spread over $N$ excited levels $\left|\beta^{\prime \prime} J^{\prime \prime}\right\rangle$, which means that $w_{\beta^{\prime \prime} m}^{\left(J^{\prime \prime}\right)}=1 / N$ for all $J^{\prime \prime}$, then Eq. (38) is $1 / N$, and Eq. (39) is divided by $N$.

(ii) It tends to enhance the vector $\operatorname{Im}\left[\alpha_{1}^{6 p}(\omega)\right]$ and tensor $\operatorname{Im}\left[\alpha_{2}^{6 p}(\omega)\right]$ contributions, because for arbitrary weights (different from 0,1 , and $1 / N)$, the three $J^{\prime \prime}$ terms in Eq. (37) do not exactly compensate each other.

The weights $w_{\beta^{\prime \prime} m}^{\left(J^{\prime \prime}\right)}$ associated with the eigenvectors of excited energy levels are therefore crucial for calculation of the imaginary part of the polarizability. In our previous work on erbium [45], we described the odd-parity levels with the configurations $4 f^{12} 6 s 6 p, 4 f^{11} 5 d 6 s^{2}$, and $4 f^{11} 5 d^{2} 6 s$, which is likely to yield a reliable calculation of the weights $w_{\beta^{\prime \prime}, 1}^{\left(J^{\prime \prime}\right)}$, which play an important part in the polarizability. By contrast, we did not consider the configurations $4 f^{q-1} 5 d^{2} 6 s$ for Dy $(q=10)$ and Ho $(q=11)$, because of the large number of levels belonging to these configurations. Since some of the weights $w_{\beta^{\prime \prime} m}^{\left(J^{\prime \prime}\right)}$ are not correct, our computed imaginary polarizabilities must be taken with caution. The relatively low 
ratio of the vector and tensor contributions with respect to the scalar one, observed in Ref. [46] and Sec. III C, may be due to the lack of $\mathrm{CI}$ in our eigenvectors. In the next section, we present a method to estimate the weights $w_{\beta^{\prime \prime}, 1}^{\left(J^{\prime \prime}\right)}$ from experimental values of the transition probabilities.

\section{Estimate of configuration-interaction mixing}

We consider transition probabilities $A_{\beta^{\prime \prime} J^{\prime \prime}, J 0}$, characterizing the spontaneous emissions from level $\left|\beta^{\prime \prime} J^{\prime \prime}\right\rangle$ towards the ground level $|0 J\rangle$, which are given by Eq. (27) with $|\tilde{\beta} \tilde{J}\rangle=|0 J\rangle$. Assuming that the transition is due to the coupling between basis states $\left|1 J^{\prime \prime}\right\rangle$ and $|0 J\rangle$, we obtain that the squared transition dipole moment is proportional to $w_{\beta^{\prime \prime}, 1}^{\left(J^{\prime \prime}\right)}$ [see Eq. (33)], and so the Einstein coefficient is proportional to $A_{\beta^{\prime \prime} J^{\prime \prime}, 0 J} \propto w_{\beta^{\prime \prime}, 1}^{\left(J^{\prime \prime}\right)} \times\left(E_{\beta^{\prime \prime} J^{\prime \prime}}-E_{0 J}\right)^{3}$. Supposing all transition energies approximately equal, i.e., $E_{\beta^{\prime \prime} J^{\prime \prime}}-E_{0 J} \approx \hbar \omega_{6 p}$, yields that the sum of the transition probabilities for a given $J$ and $J^{\prime \prime}$ is a $J$ - and $J^{\prime \prime}$-independent constant,

$$
\sum_{\beta^{\prime \prime}} A_{\beta^{\prime \prime} J^{\prime \prime}, 0 J} \approx \frac{2 \omega_{6 p}^{3} e^{2} r_{6 s, 6 p}^{2}}{3 \pi \epsilon_{0} \hbar c^{3}}
$$

Therefore, knowing the transition energies and transition probabilities, we can express the weight $w_{\beta^{\prime \prime}, 1}^{\left(J^{\prime \prime}\right)}$ as

$$
w_{\beta^{\prime \prime}, 1}^{\left(J^{\prime \prime}\right)}=\frac{\frac{A_{\beta^{\prime \prime} J^{\prime \prime}, 0 J}}{\left(E_{\beta^{\prime \prime} J^{\prime \prime}}-E_{0 J}\right)^{3}}}{\sum_{\beta^{*}} \frac{A_{\beta^{*} J^{\prime \prime}, 0 J}}{\left(E_{\beta^{*} J^{\prime \prime}}-E_{0 J}\right)^{3}}},
$$

where the terms $\left(E_{\beta^{\prime \prime} J^{\prime \prime}}-E_{0 J}\right)$ and $\left(E_{\beta^{*} J^{\prime \prime}}-E_{0 J}\right)$ have been explicitly written, in order to get a better estimate of $w_{\beta^{\prime \prime}, 1}^{\left(J^{\prime \prime}\right)}$, even though they could be approximated by $\hbar \omega_{6 p}$.

In practice, Lawler and Den Artog's group performed extensive measurements of transition probabilities, especially in dysprosium [55], holmium [56], erbium [57], and thulium [58]. The spectrum of the ground level is composed of a forest of weak transitions from which emerge a few strong transitions with similar transition energies. The number of strong lines (say with $A_{\beta^{\prime \prime} J^{\prime \prime}, 0 J}>10^{7} \mathrm{~s}^{-1}$ ) increases with increasing atomic number. When calculating the sum of Einstein coefficients for separated $J^{\prime \prime}$ [see Eq. (40)], one usually finds 2.1 to $2.4 \times 10^{8} \mathrm{~s}^{-1}$. Among these transitions, some are certainly due not to $6 s-6 p$ but, rather, to $4 f-5 d$ excitation; however, they are so weak that they will not affect the calculation of $w_{\beta^{\prime \prime}, 1}^{\left(J^{\prime \prime}\right)}$ with Eq. (41).

In the case of erbium, we modeled the erbium spectrum including configurations $4 f^{12} 6 s 6 p, 4 f^{11} 5 d 6 s^{2}$, and $4 f^{11} 5 d^{2} 6 s$ [45], while we did not include either $4 f^{9} 5 d^{2} 6 s$ for dysprosium [46] or $4 f^{10} 5 d^{2} 6 s$ for holmium (see Sec. III). So for erbium, the "experimental" weights, given by Eq. (41), can be compared with the "theoretical" ones, which we can extract from our modeling of the spectrum [45]. The results are listed in Table I for the odd-parity levels giving the strongest transitions (with probabilities higher than $10^{7} \mathrm{~s}^{-1}$ ) towards the ground level $4 f^{12} 6 s^{2}{ }^{3} H_{6}$. In Table I we also compare the energies and transition probabilities. As discussed in Ref. [45], the agreementfor the energy is very good. As for the transition probabilities, the overall agreement is satisfactory, even if the theoretical transition probabilities and weights are globally
TABLE I. Comparison of theoretical and experimental energies of selected excited odd-parity levels $\left|\beta^{\prime \prime} J^{\prime \prime}\right\rangle$ of erbium, of transition probabilities characterizing the spontaneous emission from levels $\left|\beta^{\prime \prime} J^{\prime \prime}\right\rangle$ to the ground level $|0 J\rangle=\left|4 f^{12} 6 s^{23} H_{6}\right\rangle$, and of the weight of the component $\left|1 J^{\prime \prime}\right\rangle=\left|4 f^{12}\left({ }^{3} H_{6}\right) \cdot 6 s 6 p\left({ }^{1} P_{1}^{o}\right)(6,1)_{J^{\prime \prime}}^{o}\right\rangle$ in the eigenvector associated with level $\left|\beta^{\prime \prime} J^{\prime \prime}\right\rangle$ [see Eq. (32)]. Theoretical quantities, in the columns "Theor.," come from our previous work [45], whereas experimental ones, in the columns "Expt.," come from Ref. [57]. Experimental weights $w_{\beta^{\prime \prime}, 1}^{\left(J^{\prime \prime}\right)}$ are given by Eq. (41). The selected excited levels are such that the experimental transition probability towards the ground level is higher than $10^{7} \mathrm{~s}^{-1}$. Values in

\begin{tabular}{|c|c|c|c|c|c|c|}
\hline \multicolumn{2}{|c|}{$E_{\beta^{\prime \prime} J^{\prime \prime}}\left(\mathrm{cm}^{-1}\right)$} & \multirow[b]{2}{*}{$J^{\prime \prime}$} & \multicolumn{2}{|c|}{$A_{\beta^{\prime \prime} J^{\prime \prime}, 0 J}\left(\mathrm{~s}^{-1}\right)$} & \multicolumn{2}{|c|}{$w_{\beta^{\prime \prime}, 1}^{\left(J^{\prime \prime}\right)}(\%)$} \\
\hline Expt. & Theor. & & Expt. & Theor. & Expt. & Theor. \\
\hline 24083 & 24056 & 5 & $1.02(8)$ & $9.34(7)$ & 48 & 46 \\
\hline 24457 & 24492 & 6 & $3.26(7)$ & $2.16(7)$ & 16 & 11 \\
\hline 24943 & 24946 & 7 & $1.85(8)$ & $2.08(8)$ & 76 & 79 \\
\hline 25159 & 25168 & 7 & $4.03(7)$ & $1.27(7)$ & 16 & 5 \\
\hline 25163 & 25171 & 5 & $3.76(7)$ & $4.60(7)$ & 15 & 16 \\
\hline 25393 & 25419 & 6 & $3.19(7)$ & $1.86(7)$ & 14 & 7 \\
\hline 25598 & 25570 & 7 & $1.51(7)$ & $5.50(6)$ & 6 & 2 \\
\hline 25682 & 25598 & 5 & $6.3(7)$ & $4.28(7)$ & 24 & 13 \\
\hline 25880 & 26071 & 6 & $1.22(8)$ & $9.68(7)$ & 49 & 31 \\
\hline 26237 & 26178 & 6 & $2.90(7)$ & $8.43(7)$ & 11 & 26 \\
\hline
\end{tabular}
parentheses $(n)$ indicate $\times 10^{n}$.

lower than the experimental ones. For a given level $\left|\beta^{\prime \prime} J^{\prime \prime}\right\rangle$, the discrepancies in $A_{\beta^{\prime \prime} J^{\prime \prime}, 0 J}$ and for $w_{\beta^{\prime \prime}, 1}^{\left(J^{\prime \prime}\right)}$ are actually similar. This confirms our assumption that the strongest transitions are due to the $\left|1 J^{\prime \prime}\right\rangle \rightarrow|0 J\rangle$ components. This also means that, taking the experimental transition probabilities as benchmarks, we may improve our theoretical values by improving the quality of our eigenvectors.

To illustrate the validity of our weight calculations, in Table II, we list the real part of the scalar contribution, as well as the imaginary part of the scalar, vector, and tensor contributions of the dynamic dipole polarizability at the frequency corresponding to a 1064-nm wavelength, for erbium, holmium, and dysprosium. The calculations are carried out using three methods. (i) The transition energies and squares of the transition dipole moments are taken from our full numerical modeling of the atomic spectra. In particular, the squares of the transition dipole moments are extracted from the Einstein coefficients, by reversing Eq. (27):

$$
\left|\left\langle\beta^{\prime \prime} J^{\prime \prime}\|\mathbf{d}\| 0 J\right\rangle\right|^{2}=\frac{3 \pi \epsilon_{0} \hbar^{4} c^{3}\left(2 J^{\prime \prime}+1\right) A_{\beta^{\prime \prime} J^{\prime \prime}, 0 J}}{\left(E_{\beta^{\prime \prime} J^{\prime \prime}}-E_{0 J}\right)^{3}} .
$$

This corresponds to the columns entitled "Theor." in Table II. (ii) The transition energies and the squares of the transition dipole moments come from experimental measurements of the transition probabilities using Eq. (42); this corresponds to the columns entitled "Expt." in Table II. (iii) Polarizabilities are calculated using Eqs. (20) and (21); to that end, the weights $w_{\beta^{\prime \prime}, 1}^{\left(J^{\prime \prime}\right)}$ are calculated by applying Eq. (41) with experimental data, and the quantities $r_{6 s 6 p}$ come from our fitting procedure of Einstein coefficients, namely, $r_{6 s 6 p}=3.551$ a.u. for $\operatorname{Er}$ [45], 3.648 a.u. for Dy [46], and 3.630 a.u. for Ho (see Sec. III; for dipole moments, 1 a.u. $=e a_{0}$, with $a_{0}$ the Bohr radius). This 
TABLE II. Dynamic dipole polarizabilities of dysprosium, holmium, and erbium in their ground level, at the commonly used 1064-nm trapping wavelength. Namely, we give the real part of the scalar contribution (in atomic units; 1 a.u. $=e^{2} a_{0}^{3} / 4 \pi \epsilon_{0}$ ), as well as the imaginary part of the scalar, vector, and tensor contributions (in $10^{-7}$ a.u.). The last two lines list the vector-to-scalar and tensor-to-scalar ratios of the imaginary part. The columns "Theor." and "Expt." list the theoretical (see Refs. [45] and [46] and Sec. III C here) and experimental [55-57] transition energies and transition probabilities respectively. The columns "Eq. (41)" correspond to the application of Eqs. (20), (21), and (41).

\begin{tabular}{|c|c|c|c|c|c|c|c|c|c|c|}
\hline \multirow[b]{2}{*}{ Part } & \multirow[b]{2}{*}{ Contribution } & \multicolumn{3}{|c|}{ Dy $\left({ }^{5} I_{8}\right)$} & \multicolumn{3}{|c|}{ Ho $\left({ }^{4} I_{15 / 2}^{o}\right)$} & \multicolumn{3}{|c|}{$\operatorname{Er}\left({ }^{3} H_{6}\right)$} \\
\hline & & Theor. & Expt. & Eq. (41) & Theor. & Expt. & Eq. (41) & Theor. & Expt. & Eq. (41) \\
\hline Real & Scalar & 193 & 177 & 188 & 187 & 160 & 186 & 164 & 155 & 170 \\
\hline \multirow[t]{3}{*}{ Imaginary } & Scalar & 49.1 & 40.3 & 48.8 & 39.6 & 34.7 & 46.6 & 23.4 & 22.0 & 27.1 \\
\hline & Vector & 11.3 & 12.9 & 15.2 & 19.1 & 17.0 & 17.1 & 17.4 & 11.2 & 12.4 \\
\hline & Tensor & 5.8 & -9.0 & -11.3 & 4.9 & 5.5 & 9.2 & -6.9 & -5.4 & -5.0 \\
\hline $\begin{array}{l}\text { Vector/ } \\
\text { scalar ratio }\end{array}$ & & 0.230 & 0.320 & 0.311 & 0.482 & 0.490 & 0.367 & 0.744 & 0.509 & 0.458 \\
\hline $\begin{array}{l}\text { Tensor/ } \\
\text { scalar ratio }\end{array}$ & & 0.118 & -0.223 & -0.244 & 0.124 & 0.159 & 0.197 & -0.295 & -0.245 & -0.185 \\
\hline
\end{tabular}

corresponds to the columns entitled "Eq. (41)" in Table II. The real parts of the vector and tensor contributions are pointless here, as they vanish with method (iii).

First, we see that the real part of the scalar polarizability is smaller with the Expt. method. This is particularly striking in the case of holmium. In comparison with the Theor. method, this is due to the smaller number of experimental transitions than of theoretical ones. In contrast, the number of transitions in the Expt. and Eq. (41) methods is the same; however, we saw in Table I that the experimental weights are overestimated. Indeed there are certainly transitions with upper levels having a small $\left|1 J^{\prime \prime}\right\rangle$ character which have not been detected. This results in the underestimation of the denominator of Eq. (41) and, so, the overestimation of $w_{\beta^{\prime \prime}, 1}^{\left(J^{\prime \prime}\right)}$. Similar discrepancies are visible for the imaginary part of the scalar polarizability. Therefore it is appropriate to analyze the vector-to-scalar and tensor-to-scalar ratio contributions, in order to determine the anisotropy of the photon-scattering rate. The overall agreement of those ratios is good, and the two following trends are visible with the three methods: (i) In absolute value, the vector-toscalar ratios are higher than the tensor-to-scalar ones; and (ii) the ratios are higher for erbium than for holmium, and $a$ fortiori for dysprosium. Beyond these general features, it is worthwhile to examine each atom separately.

Erbium is the atom for which the anisotropy is the most pronounced, for both the vector and the tensor contributions, even if the ratios vary significantly from one method to the other. From the Eq. (41) method to the Theor. method, the ratios $\operatorname{Im}\left(\alpha_{\text {vect }}\right) / \operatorname{Im}\left(\alpha_{\text {scal }}\right)$ and $\operatorname{Im}\left(\alpha_{\text {tens }}\right) / \operatorname{Im}\left(\alpha_{\text {scal }}\right)$ range from 0.458 and -0.185 to 0.744 and -0.295 , respectively.

In the case of dysprosium, the agreement between the Expt. and the Eq. (41) methods is very good. The ratios $\operatorname{Im}\left(\alpha_{\text {vect }}\right) / \operatorname{Im}\left(\alpha_{\text {scal }}\right)$ are equal to 0.320 and 0.311 , and the ratios $\operatorname{Im}\left(\alpha_{\text {tens }}\right) / \operatorname{Im}\left(\alpha_{\text {scal }}\right)$ to -0.223 and -0.244 , respectively. With the Theor. method, the ratios are lower, $\left(\operatorname{Im}\left(\alpha_{\text {vect }}\right) / \operatorname{Im}\left(\alpha_{\text {scal }}\right)=\right.$ 0.230 and $\left.\operatorname{Im}\left(\alpha_{\text {tens }}\right) / \operatorname{Im}\left(\alpha_{\text {scal }}\right)=0.118\right)$, especially because this method does not allow for describing the CI mixing in the levels at 23832 and $23878 \mathrm{~cm}^{-1}$, and so it underestimates Eq. (38).

Finally, the case of holmium is hard to analyze, since no particular trend comes out of the calculations. The real part of the scalar polarizability is 27 a.u. smaller in the Expt. method than in the two others. Moreover, regarding the experimental transitions towards the ground level, none of them imply an upper level with an energy above $25571 \mathrm{~cm}^{-1}$. These two facts suggest the possibility that some strong transitions have not been detected, especially with upper levels $J^{\prime \prime}=13 / 2$. For instance, in our full numerical modeling of the Ho spectrum (see Sec. III B) we predict two such transitions, with unobserved upper levels: one with $E_{\beta^{\prime \prime}, 13 / 2}^{\text {th }}=28014 \mathrm{~cm}^{-1}, w_{\beta^{\prime \prime}, 1}^{(13 / 2)}=4 \%$, $A_{\beta^{\prime \prime}, 13 / 2,0 J}^{\text {th }}=2.61 \times 10^{7} \mathrm{~s}^{-1}$ and the other with $E_{\beta^{\prime \prime}, 13 / 2}^{\text {th }}=$ $30942 \mathrm{~cm}^{-1}, w_{\beta^{\prime \prime}, 1}^{(13 / 2)}=7 \%, A_{\beta^{\prime \prime}, 13 / 2,0 J}^{\text {th }}=1.89 \times 10^{7} \mathrm{~s}^{-1}$.

\section{MODELING OF THE HOLMIUM SPECTRUM}

In order to calculate the different components of the polarizabilities, and also the various $C_{6}$ coefficients, using the sum-over-state formulas, one needs an extensive set of transition energies and transition dipole moments. This section is devoted to the full numerical calculations of those quantities, in the case of holmium in its ground ${ }^{4} I_{15 / 2}^{o}$ and first excited level ${ }^{4} I_{13 / 2}^{o}$. Indeed the transition between those two levels, allowed in the electric-quadrupole and magnetic-dipole approximations, was suggested as a candidate for optical clocks $[19,21]$, as these levels are expected to possess very similar polarizabilities.

As the principle of our calculations [47-49] is identical to that in our previous work on dysprosium [46], we only highlight in this section the particularities of holmium. One of them is the rarity of experimental Landé $g$ factors, which gives to our work a predictive character in this respect. The experimental energies are published in the NIST database [54], constructed from the critical compilation of Martin et al. [59] and from Ref. [60], which is more recent than the compilation. For odd-parity levels, we also use unpublished work from our group [61]. Note that ${ }^{165} \mathrm{Ho}$, which is bosonic, possesses a nuclear spin $I=7 / 2$, but the resulting hyperfine structure is not considered in the present article.

\section{A. Energy levels}

The ground level of holmium is of odd parity, with the configuration $4 f^{11} 6 s^{2}$ and total electronic angular momentum $J=15 / 2$. Table III presents a comparison of our 
TABLE III. Comparison of energies $E$ through the quantity $\Delta E=E^{\text {expt }}-E^{\text {th }}$ and Landé $g$ factors $g_{L}$ of Ho I odd-parity levels of the lowest electronic configuration [Xe] $4 f^{11} 6 s^{2}$. The superscript "expt" indicates experimental values, which are taken from [54] and [60]. The superscript "th" indicates theoretical values from the parametric study in Ref. [61].

\begin{tabular}{lllcclc}
\hline \hline Term & $J$ & $\begin{array}{l}E^{\text {expt }} \\
\left(\mathrm{cm}^{-1}\right)\end{array}$ & $\begin{array}{l}\Delta E \\
\left(\mathrm{~cm}^{-1}\right)\end{array}$ & $g_{L}^{\text {expt }}$ & $g_{L}^{\text {th }}$ & $\begin{array}{c}\text { \% leading } \\
\text { term }\end{array}$ \\
\hline${ }^{4} \mathrm{I}^{o}$ & $15 / 2$ & 0 & 30 & 1.195 & 1.197 & 97 \\
${ }^{4} I^{o}$ & $13 / 2$ & 5419.70 & 7 & - & 1.107 & 99 \\
${ }^{4} I^{o}$ & $11 / 2$ & 8605.16 & -6 & 1.012 & 0.985 & 85 \\
${ }^{4} I^{o}$ & $9 / 2$ & 10695.75 & -5 & 0.866 & 0.864 & 60 \\
${ }^{4} F^{o}$ & $9 / 2$ & 13094.42 & 46 & - & 1.174 & 65 \\
${ }^{4} G^{o}$ & $11 / 2$ & 22593.53 & -90 & - & 1.193 & 44 \\
\hline \hline
\end{tabular}

theoretical energies and Landé $g$ factors versus their experimental counterparts. The theoretical values are obtained in a calculation including the configurations $4 f^{11} 6 s^{2}, 4 f^{11} 5 d 6 s$, and $4 f^{10} 6 s^{2} 6 p$ [61]. The levels of the $4 f^{11} 6 s^{2}$ configuration can be labeled in the $L S$ coupling scheme; for example, the orbital $L=6$ and spin $S=3 / 2$ angular momenta of the ground level are good quantum numbers up to $97 \%$. By contrast, the level at $22593.53 \mathrm{~cm}^{-1}$ is of ${ }^{4} G$ and ${ }^{2} H$ characters up to $44 \%$ and $36 \%$, respectively

In the even parity, the electronic configurations included in our model are the two lowest ones, $4 f^{11} 6 s 6 p$ and $4 f^{10} 5 d 6 s^{2}$ [62], which are connected to the ground-state configuration $4 f^{11} 6 s^{2}$ by electric-dipole transitions. Therefore, in our model, we neglect the configuration interaction with other even-parity configurations, especially $4 f^{10} 5 d^{2} 6 s$, which contains a large number of levels. By contrast, the first parametric study of even-parity levels was performed with configurations with a limited number of $L S$ terms of the $4 f^{10}$ and $4 f^{11}$ cores, including a configuration interaction with $4 f^{10} 5 d^{2} 6 s$; but such a truncation strongly damaged the quality of the Hamiltonian eigenvectors [63]. In the present study, 92 even-parity levels were fitted to their known experimental counterparts $[54,60]$, using 21 free energetic parameters, giving a $45-\mathrm{cm}^{-1}$ standard deviation.

A comparison between theoretical and experimental levels is reported in Table VIII, while the fitted parameters are listed in Table IX (see the Appendix). Due to the lack of experimental $g$-factor data for most levels, we just list the theoretical results. All energies are given relative to the experimental $4 f^{11} 6 s^{24} I_{15 / 2}^{o}$ ground level. Despite the absence of the $4 f^{10} 5 d^{2} 6 s$ configuration, whose lowest classified level is at $20167.17 \mathrm{~cm}^{-1}$, the agreement is very satisfactory.

\section{B. Transition probabilities}

Now that the energy parameters have been adjusted, the eigenvalues and eigenvectors of the Hamiltonian operator are fixed. The transition probabilities also depend on the MTDMs $-e r_{n \ell, n^{\prime \prime} \ell^{\prime \prime}}$, whose adjustment using least-squares fitting between theoretical and experimental transition probabilities is the goal of this subsection.

Due to the configurations that we consider, two MTDMs come into play, $r_{656 p}$ and $r_{4 f 5 d}$, corresponding, respec- tively, to the couples of configurations $4 f^{11} 6 s^{2}-4 f^{11} 6 s 6 p$ and $4 f^{11} 6 s^{2}-4 f^{10} 5 d 6 s^{2}$. The least-squares fitting procedure between theoretical and experimental Einstein coefficients is performed on the scaling factors (SFs) $f_{1}=r_{6 s 6 p} / r_{6 s 6 p}^{\mathrm{HFR}}$ and $f_{2}=r_{4 f 5 d} / r_{4 f 5 d}^{\mathrm{HFR}}$, rather than the MTDMs themselves. This allows for more direct comparisons with the results for dysprosium and erbium. Note that $r_{n \ell, n^{\prime \prime} \ell^{\prime \prime}}^{\mathrm{HFR}}$ stands for the $a b$ initio values calculated with the Hartree-Fock method including relativistic corrections (HFR).

As references, we take the measured transition probabilities in Ref. [56]. We retain the transitions involving the ground and first excited levels and upper levels with energies lower than $30000 \mathrm{~cm}^{-1}$. Indeed the levels above $30000 \mathrm{~cm}^{-1}$ are hard to classify unambiguously in configurations $4 f^{10} 5 d 6 s^{2}$ and $4 f^{11} 6 s 6 p$. In addition, in the list in Ref. [56], we can see some strong transitions whose upper level does not belong to the $4 f^{11} 6 s 6 p$ or $4 f^{10} 5 d 6 s^{2}$ configuration (according to the NIST database [54]), e.g., $E_{\beta^{\prime \prime} J^{\prime \prime}}^{\text {ext }}=24263.88 \mathrm{~cm}^{-1}, J^{\prime \prime}=17 / 2$, but is very close in energy to a $4 f^{11} 6 s 6 p$ level with the same $J^{\prime \prime}$, e.g., $E_{\beta^{\prime \prime} J^{\prime \prime}}^{\text {expt }}=24360.81 \mathrm{~cm}^{-1}$. In contrast there is only one close theoretical level predicted, $E_{\beta^{\prime \prime} J^{\prime \prime}}^{\text {th }}=24354.1 \mathrm{~cm}^{-1}$. Similarly to the case for dysprosium, we can assume that the eigenvector of that theoretical level contains some components of the $\left|1 J^{\prime \prime}\right\rangle$ state which is shared by the two "real" levels. In these particular cases, we compare our theoretical Einstein coefficient with the sum of the experimental ones. In Table IV, the two transitions labeled "mixed" correspond to that situation.

Due to strong differences between experimental and theoretical Einstein coefficients, we excluded six transitions (one with a high ratio, $A_{\beta^{\prime \prime} J^{\prime \prime}, \beta J}^{\text {th }} / A_{\beta^{\prime \prime} J^{\prime \prime}, \beta J}^{\exp }$, and another four with

TABLE IV. Transitions excluded from the least-squares fitting procedure. The labels $\left|\beta^{\prime \prime} J^{\prime \prime}\right\rangle$ and $|\beta J\rangle$ correspond to upper and lower levels, respectively. The superscript "expt" indicates experimental values, which are taken from [56]. The transition wave number $\sigma_{\beta^{\prime \prime} J^{\prime \prime}, \beta J}=\left(E_{\beta^{\prime \prime} J^{\prime \prime}}-E_{\beta}\right) / 2 \pi \hbar c$ is in the vacuum. Values in parentheses $(n)$ indicate $\times 10^{n}$. A blank in the column "removal reason" indicates that the upper level belongs neither to the $4 f^{10} 6 s 6 p$ nor to the $4 f^{9} 5 d 6 s^{2}$ configuration. r., ratio.

\begin{tabular}{lcccccc}
\hline \hline $\begin{array}{l}E_{\beta^{\prime \prime} J^{\prime \prime}}^{\text {expt }} \\
\left(\mathrm{cm}^{-1}\right)\end{array}$ & $J^{\prime \prime}$ & $\begin{array}{l}E_{\beta J}^{\text {exp }} \\
\left(\mathrm{cm}^{-1}\right)\end{array}$ & $J$ & $\begin{array}{l}\sigma_{\beta^{\prime \prime} J^{\prime \prime} \beta J}^{\text {expt }} \\
\left(\mathrm{cm}^{-1}\right)\end{array}$ & $\begin{array}{l}A_{\beta^{\prime \prime} J^{\prime \prime}, \beta J}^{\text {expt }} \\
\left(\mathrm{s}^{-1}\right)\end{array}$ & $\begin{array}{l}\text { Removal } \\
\text { reason }\end{array}$ \\
\hline 20258 & 6.5 & 0 & 7.5 & 20258 & $3.40(5)$ & \\
24014 & 6.5 & 0 & 7.5 & 24014 & $1.06(8)$ & Large r. \\
24264 & 8.5 & 0 & 7.5 & 24264 & $1.42(7)$ & Mixed \\
24377 & 7.5 & 0 & 7.5 & 24377 & $5.78(6)$ & Mixed \\
24760 & 6.5 & 0 & 7.5 & 24760 & $1.20(6)$ & \\
17059 & 6.5 & 5420 & 6.5 & 11640 & $0.34(3)$ & Large r. \\
18756 & 7.5 & 5420 & 6.5 & 13337 & $1.92(4)$ & Small r. \\
18858 & 6.5 & 5420 & 6.5 & 13438 & $0.91(4)$ & Small r. \\
20258 & 6.5 & 5420 & 6.5 & 14839 & $0.42(5)$ & \\
24760 & 6.5 & 5420 & 6.5 & 19340 & $0.47(4)$ & \\
25571 & 6.5 & 5420 & 6.5 & 20151 & $0.38(5)$ & Small r. \\
20241 & 6.5 & 8605 & 5.5 & 11636 & $1.19(4)$ & Small r. \\
20258 & 6.5 & 8605 & 5.5 & 11653 & $0.20(4)$ & \\
22978 & 6.5 & 8605 & 5.5 & 14373 & $4.28(4)$ & Small r. \\
24760 & 6.5 & 8605 & 5.5 & 16155 & $0.48(4)$ & \\
\hline \hline
\end{tabular}

${ }^{\text {a }}$ Mixed with level at $24361 \mathrm{~cm}^{-1}$.

${ }^{\mathrm{b}}$ Mixed with level at $24661 \mathrm{~cm}^{-1}$. 
TABLE V. Comparison of Einstein A coefficients. The superscript "expt" indicates experimental values, which are taken from [56]. The superscript "th" indicates theoretical values from the present calculations. Values in parentheses $(n)$ indicate $\times 10^{n}$. Values followed by an asterisk correspond to sums of experimental Einstein coefficients (see Table IV).

\begin{tabular}{|c|c|c|c|c|c|c|}
\hline $\begin{array}{l}E_{\beta^{\prime \prime} J^{\prime \prime}}^{\operatorname{expt}} \\
\left(\mathrm{cm}^{-1}\right)\end{array}$ & $J^{\prime \prime}$ & $\begin{array}{c}E_{\beta J}^{\text {expt }} \\
\left(\mathrm{cm}^{-1}\right)\end{array}$ & $J$ & $\begin{array}{l}\sigma_{\beta^{\prime \prime} J^{\prime \prime}, \beta J}^{\operatorname{expt}} \\
\left(\mathrm{cm}^{-1}\right)\end{array}$ & $\begin{array}{l}A_{\beta^{\prime \prime} J^{\prime \prime}, \beta J}^{\text {expt }} \\
\left(\mathrm{s}^{-1}\right)\end{array}$ & $\begin{array}{c}A_{\beta^{\prime \prime} J^{\prime \prime}, \beta J}^{\mathrm{th}} \\
\quad\left(\mathrm{s}^{-1}\right)\end{array}$ \\
\hline 16710 & 8.5 & 0 & 7.5 & 16710 & $9.20(5)$ & $1.51(6)$ \\
\hline 16882 & 7.5 & 0 & 7.5 & 16882 & $3.60(5)$ & 7.91(5) \\
\hline 17059 & 6.5 & 0 & 7.5 & 17059 & $6.50(5)$ & $1.25(6)$ \\
\hline 18652 & 7.5 & 0 & 7.5 & 18652 & $2.99(5)$ & $2.19(5)$ \\
\hline 18756 & 7.5 & 0 & 7.5 & 18756 & $2.20(5)$ & $5.77(4)$ \\
\hline 18858 & 6.5 & 0 & 7.5 & 18858 & $2.70(5)$ & $2.87(5)$ \\
\hline 20075 & 7.5 & 0 & 7.5 & 20075 & $1.11(6)$ & $6.24(5)$ \\
\hline 20241 & 6.5 & 0 & 7.5 & 20241 & $2.15(6)$ & $1.72(6)$ \\
\hline 22978 & 6.5 & 0 & 7.5 & 22978 & $9.30(6)$ & $1.18(7)$ \\
\hline 23445 & 7.5 & 0 & 7.5 & 23445 & $3.70(6)$ & $3.62(6)$ \\
\hline 23499 & 8.5 & 0 & 7.5 & 23499 & $1.00(7)$ & $1.13(7)$ \\
\hline 23835 & 7.5 & 0 & 7.5 & 23835 & $3.88(6)$ & $2.79(6)$ \\
\hline 23956 & 6.5 & 0 & 7.5 & 23956 & $3.12(7)$ & $2.23(7)$ \\
\hline 24361 & 8.5 & 0 & 7.5 & 24361 & $2.18(8)^{*}$ & $2.18(8)$ \\
\hline 24661 & 7.5 & 0 & 7.5 & 24661 & $2.06(8)^{*}$ & $2.14(8)$ \\
\hline 24741 & 6.5 & 0 & 7.5 & 24741 & $4.48(7)$ & $3.08(7)$ \\
\hline 25273 & 7.5 & 0 & 7.5 & 25273 & $6.30(6)$ & $8.32(6)$ \\
\hline 25571 & 6.5 & 0 & 7.5 & 25571 & $5.24(5)$ & $5.08(5)$ \\
\hline 16882 & 7.5 & 5420 & 6.5 & 11463 & $6.00(3)$ & $4.92(3)$ \\
\hline 20075 & 7.5 & 5420 & 6.5 & 14655 & $5.40(4)$ & $7.48(3)$ \\
\hline 20241 & 6.5 & 5420 & 6.5 & 14822 & $2.58(5)$ & $3.38(4)$ \\
\hline 22413 & 5.5 & 5420 & 6.5 & 16993 & $8.70(5)$ & $1.51(6)$ \\
\hline 22978 & 6.5 & 5420 & 6.5 & 17558 & $2.60(5)$ & $3.94(4)$ \\
\hline 24741 & 6.5 & 5420 & 6.5 & 19321 & $3.52(5)$ & $8.96(4)$ \\
\hline 25273 & 7.5 & 5420 & 6.5 & 19853 & $2.99(4)$ & $1.82(4)$ \\
\hline 29070 & 5.5 & 5420 & 6.5 & 23650 & $1.06(8)$ & 1.11(8) \\
\hline 29097 & 5.5 & 5420 & 6.5 & 23677 & $6.70(6)$ & $2.80(6)$ \\
\hline 29643 & 7.5 & 5420 & 6.5 & 24223 & $2.12(8)$ & $2.13(8)$ \\
\hline 29752 & 6.5 & 5420 & 6.5 & 24332 & $2.00(8)$ & $1.92(8)$ \\
\hline 25571 & 6.5 & 8605 & 5.5 & 16966 & $7.50(5)$ & $1.39(6)$ \\
\hline
\end{tabular}

very low ratios). Special attention should be paid to the strong transition between the ground level and the excited $J^{\prime \prime}=13 / 2$ level at $E_{\beta^{\prime \prime} J^{\prime \prime}}^{\mathrm{expt}}=24014.2 \mathrm{~cm}^{-1}$. For the optimal scaling factors $f_{1}$ and $f_{2}$ (see below), the error in the other strongest transitions (above $10^{8} \mathrm{~s}^{-1}$ ) is below $5 \%$, while for the latter it is $14 \%$. This may be due to an underestimated experimental value. Another possible explanation is the following: There is a close $J^{\prime \prime}=6.5$ level, at $E_{\beta^{\prime \prime} J^{\prime \prime}}^{\text {expt }}=23955.69 \mathrm{~cm}^{-1}$, where comparing the sum of transition probabilities implying these two upper levels, the theory-experiment agreement is very good (1.47 and $1.42 \times 10^{8} \mathrm{~s}^{-1}$, respectively). The agreement for individual transitions can probably be improved by better CI mixing between the configurations $4 f^{11} 6 s 6 p$ and $4 f^{10} 5 d 6 s^{2}$.

We fitted the SFs using the remaining 29 transitions and found optimal SFs $f_{1}=0.798$ and $f_{2}=0.969$, corresponding to a standard deviation in Einstein coefficients [see Ref. [45], Eq. (15)] $\sigma_{A}=4.14 \times 10^{6} \mathrm{~s}^{-1}$. In particular the five strongest transitions are calculated with a precision better than $5 \%$. Then, because the experimental Einstein coefficients in Ref. [56] are given with uncertainties reaching up to $10 \%$, we made 10000 fits in which all the experimental $A$ coefficients have a random value within their uncertainty range. We obtain optimal SFs with statistical uncertainties: $f_{1}=0.799 \pm 0.010$ and $f_{2}=0.97 \pm 0.24$. The standard deviation is therefore much more sensitive to $r_{6 s 6 p}$ than to $r_{4 f 5 d}$, since it involves the strongest transitions $[45,46,48]$. In what follows, we take the optimal scaling factors $f_{1}=0.799$ and $f_{2}=0.97$, for which a comparison between experimental and theoretical transition probabilities involving the two lowest levels of Ho I is presented in Table V. Using these optimal SFs, we can also calculate transition probabilities, which have not been measured and which are available upon request to the authors. In particular, as discussed in Sec. II D, we predict two strong transitions with unobserved upper levels of $J^{\prime \prime}=13 / 2$.

\section{Dynamic dipole polarizability}

The optimal set of spectroscopic data obtained in the previous subsection is now used to compute the real and imaginary parts of the scalar, vector, and tensor polarizabilities given by Eqs. (7)-(12). The squared transition dipole moments appearing in the sum are extracted from theoretical Einstein coefficients using Eq. (42).

To compare our results with the literature, the scalar, vector, and tensor static dipole polarizabilities are listed in Table VI, as well as the dynamic ones for the widespread laser-trapping 
TABLE VI. Real and imaginary parts of the scalar, vector, and tensor dynamic dipole polarizabilities, at wave numbers $\sigma=\omega / 2 \pi c=0$ and $9398 \mathrm{~cm}^{-1}$ (corresponding to $\lambda=1064 \mathrm{~nm}$ ), for the ground ${ }^{4} I_{15 / 2}^{o}$ and first excited ${ }^{4} I_{13 / 2}^{o}$ levels of holmium. Our results are compared with available literature values.

\begin{tabular}{|c|c|c|c|c|c|c|c|}
\hline \multirow[b]{2}{*}{ Level } & \multirow{2}{*}{$\begin{array}{c}\sigma \\
\left(\mathrm{cm}^{-1}\right)\end{array}$} & \multicolumn{3}{|c|}{ Real part (a.u.) } & \multicolumn{3}{|c|}{ Imaginary part $\left(10^{-7}\right.$ a.u. $)$} \\
\hline & & Scalar & Vector & Tensor & Scalar & Vector & Tensor \\
\hline${ }^{4} I_{15 / 2}^{o}$ & 0 & $\begin{array}{c}160 \\
159[65], 170[66] \\
156[67], 161[68]\end{array}$ & 0 & $\begin{array}{c}-2.3 \\
-3.19[66] \\
-1.17[69]\end{array}$ & 25.1 & 0 & 3.4 \\
\hline & 9398 & 187 & 1.1 & -3.5 & 39.6 & 19.1 & 4.9 \\
\hline${ }^{4} I_{13 / 2}^{o}$ & $\begin{array}{c}0 \\
9398\end{array}$ & $\begin{array}{l}160 \\
187\end{array}$ & $\begin{array}{c}0 \\
1.0\end{array}$ & $\begin{array}{l}-2.0 \\
-3.0\end{array}$ & $\begin{array}{l}24.1 \\
38.3\end{array}$ & $\begin{array}{c}0 \\
17.7\end{array}$ & $\begin{array}{l}1.4 \\
2.0\end{array}$ \\
\hline
\end{tabular}

wavelength $\lambda=1064 \mathrm{~nm}$ (corresponding to a wave number $\sigma=9398 \mathrm{~cm}^{-1}$ ). As one can note for the ground-level scalar polarizability, the agreement is good between the different theoretical results and with the new experimental one (for which we do not have any numerical value [64]). The tensor static polarizability is much smaller than the scalar one in all sources. For the ${ }^{4} I_{13 / 2}^{o}$ level there are no literature values to our knowledge. They are actually very similar to those of the ground level, which supports the possibility of using these levels in a clock transition.

For both levels, the main result obtained in our previous work on erbium [45] and dysprosium [46] is confirmed. Regarding the real part, the vector and tensor polarizabilities are much smaller than the scalar one. The trapping potential is thus mostly isotropic, as it hardly depends on the electric-field polarization or the atomic azimuthal quantum number. By contrast, the tensor, especially the vector contributions of the imaginary part, represent a significant fraction of the scalar contribution, which makes photon scattering anisotropic. In Sec. II D, this anisotropy is discussed in detail and compared with the results on neighboring atoms.

These features are confirmed in Figs. 1 and 2, which present the real and imaginary, respectively, parts of the scalar, vector, and tensor polarizabilities as functions of the field wavelength $\lambda$ and wave number $\sigma=1 / \lambda=\omega / 2 \pi c$ ( $c$ being the speed of light). We present our results in atomic units and the corresponding relevant quantities in physical units. The real part of the polarizability is associated with the potential energy $\bar{U}$, in equivalent temperatures of microkelvins $(\mu \mathrm{K})$, obtained for a laser intensity of $1 \mathrm{GW} / \mathrm{m}^{2}$. The imaginary part is associated with the photon-scattering rate $\bar{\Gamma}$, in inverse seconds $\left(\mathrm{s}^{-1}\right)$, for the same intensity. In Fig. 1(a), we also compare the real part of the scalar DDP given by our full numerical results and by the simplified model of Eq. (35) with $\omega_{6 p}=24320 \mathrm{~cm}^{-1}$ and $r_{6 s 6 p}=3.630$ a.u. We see that the latter reproduces very nicely the background polarizability for both levels, but not the narrow peaks due to transition toward the levels of the $4 f^{10} 5 d 6 s^{2}$ configuration or the intercombination lines toward the levels $4 f^{11}\left({ }^{4} I_{15 / 2}^{o}\right) \cdot 6 s 6 p\left({ }^{3} P_{1}^{o}\right)(15 / 2,1)_{J^{\prime \prime}}$ around $17000 \mathrm{~cm}^{-1}$.

\section{D. van der Waals $C_{6}$ coefficients}

Using the optimal spectroscopic data in Sec. III B, we can also compute the van der Waals $C_{6}$ coefficients, which also consist of a sum on the transition energies and transition dipole moments [70,71]. The van der Waals interaction between two open-shell atoms actually depends on a limited number of coefficients $C_{6, k_{A} k_{B}}$, where the indices $k_{A}$ and $k_{B}$ correspond to the rank of irreducible tensors [46,72]. Giving diagonal matrix elements, the coefficient $C_{6,00}$ is referred to as isotropic, while the other $C_{6, k_{A} k_{B}}$ are called anisotropic. Table VII lists our calculated $C_{6, k_{A} k_{B}}$ coefficients for the two lowest levels of holmium. For any pair of levels, and similarly to the case of erbium [45] and dysprosium [46], the isotropic coefficient $C_{6,00}$ strongly dominates the anisotropic ones. Moreover, the coefficients are very similar for the three pairs of levels, because the polarizabilities of levels ${ }^{4} I_{15 / 2}^{o}$ and ${ }^{4} I_{13 / 2}^{o}$ are almost equal (see Table VI).

\section{CONCLUSION}

In this article, we have derived a simplified model to characterize the optical trapping of ultracold lanthanide atoms. We have calculated analytically the real and imaginary parts of the scalar, vector, and tensor polarizabilities, assuming that the transitions involving valence electrons are the only ones present in the sum-over-state formula. We have given an analytical expression for the contribution from all the levels belonging to a given electronic configuration; this expression depends on only two parameters - an effective transition energy and an effective transition dipole moment. When applied to the two lowest levels of holmium, our model nicely reproduces the calculations based on the detailed modeling of the even-parity levels of holmium. We expect our simplified model to properly estimate the polarizabilities of levels of the $4 f^{q} 6 s 6 p$ configuration, which are relevant for trapping but which

TABLE VII. $C_{6}$ coefficients (in atomic units), characterizing the van der Waals interactions between holmium atoms in the ground ${ }^{4} I_{15 / 2}^{o}$ or first excited ${ }^{4} I_{13 / 2}^{o}$ level, as functions of the pairs of indices $k_{A}, k_{B}[46,72]$. The case $k_{A}=k_{B}=0$ corresponds to the so-called isotropic $C_{6}$ coefficient [70]. A 0 corresponds to an absolute value smaller than 0.1 a.u.

\begin{tabular}{lccc}
\hline \hline$k_{A}, k_{B}$ & ${ }^{4} I_{15 / 2}^{o}-{ }^{4} I_{15 / 2}^{o}$ & ${ }^{4} I_{15 / 2}^{o}-{ }^{4} I_{13 / 2}^{o}$ & ${ }^{4} I_{13 / 2}^{o}-{ }^{4} I_{13 / 2}^{o}$ \\
\hline 0,0 & -2214 & -2214 & -2214 \\
1,1 & 0 & 0 & 0 \\
2,0 & -8.0 & -8.0 & -7.3 \\
0,2 & -8.0 & -7.3 & -7.3 \\
2,2 & 0 & 0 & 0 \\
\hline
\end{tabular}



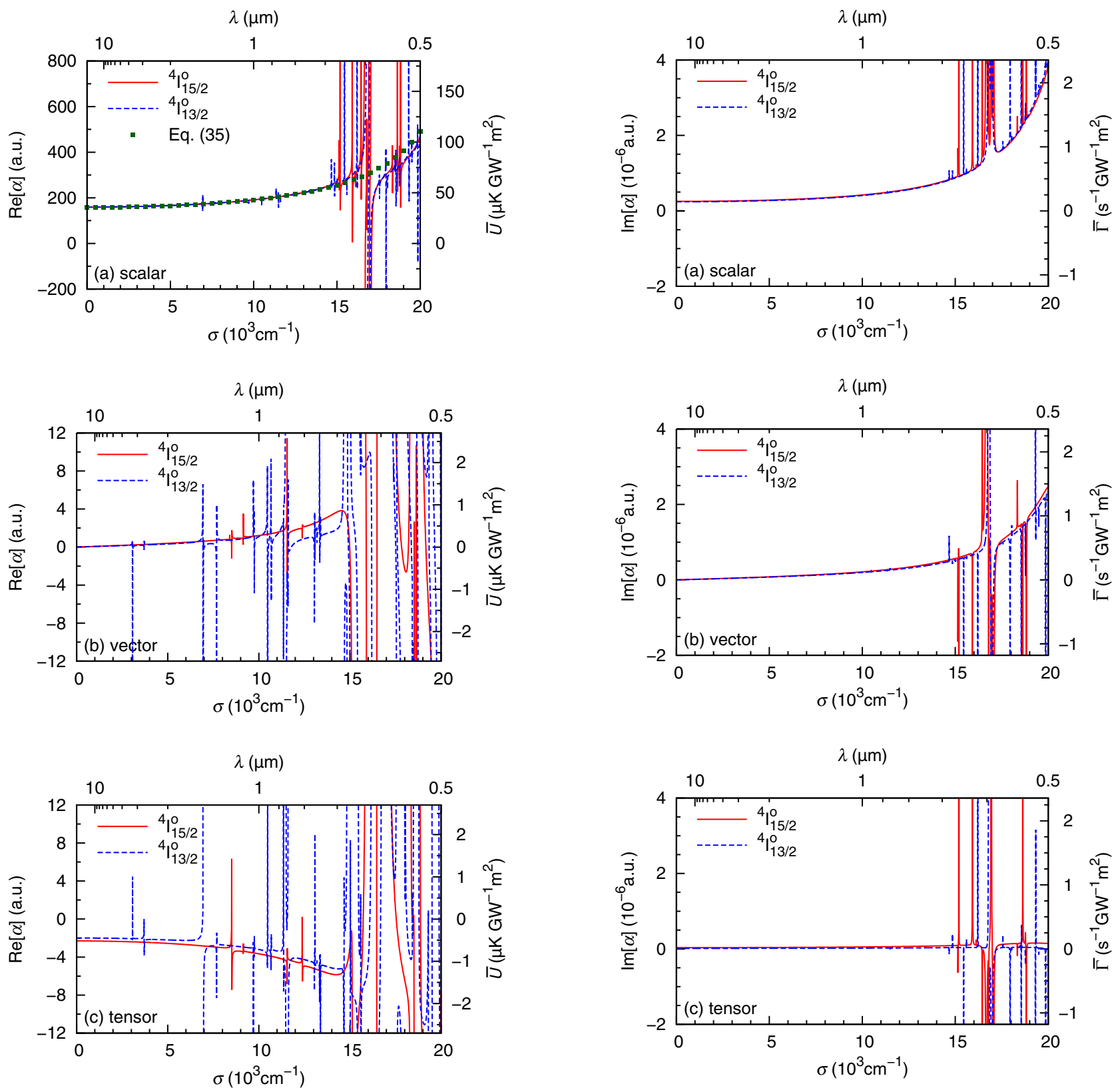

FIG. 1. Real part of the (a) scalar, (b) vector, and (c) tensor dynamic dipole polarizabilities of the ${ }^{4} I_{15 / 2}^{o}$ and ${ }^{4} I_{13 / 2}^{o}$ levels (in atomic units) and corresponding trap depths obtained for an intensity of $1 \mathrm{GW} \cdot \mathrm{m}^{-2}$, as functions of the trapping wave number $\sigma$ and wavelength $\lambda$. In (a) the real part of the scalar polarizability given by Eq. (35) is also displayed.

involve highly excited levels, for example, of configurations $4 f^{q} 6 s 6 d$ and $4 f^{q} 6 p^{2}$, rarely known experimentally. In this respect, the future study of thulium will be particularly interesting, since high-lying excited states have been characterized in detail, and so the simplified expressions given in the present paper will be compared with those involving individual transition energies and transition dipole moments.

Regarding the ground level of lanthanides, we have also studied the influence of configuration interaction between

FIG. 2. Imaginary part of the (a) scalar, (b) vector, and (c) tensor dynamic dipole polarizabilities of the ${ }^{4} I_{15 / 2}^{o}$ and ${ }^{4} I_{15 / 2}^{o}$ levels (in atomic units) and corresponding photon-scattering rates obtained for an intensity of $1 \mathrm{GW} \cdot \mathrm{m}^{-2}$, as functions of the trapping wave number $\sigma$ and wavelength $\lambda$.

$4 f^{q} 6 s 6 p$ and other configurations. We have demonstrated that the real part of the polarizability is insensitive to configuration interaction and that the vector and tensor polarizabilities are vanishingly small. By contrast, the imaginary part turns out to be very sensitive to configuration interaction among excited levels; the latter is responsible for a decrease in the scalar contribution and for an increase in the vector and tensor ones. By comparing our numerical results for dysprosium, holmium, and erbium, we have found significant variations of the imaginary part of the various polarizabilities. For example, the scalar contribution for erbium is roughly twice as small as that for dysprosium, which tends to prove that 
TABLE VIII. Same as Table III, for Ho I even-parity levels. Theoretical energies $E^{\text {th }}$, Landé factors $g_{L}^{\text {th }}$, leading configurations, leading LS terms, and their percentages in the eigenvectors, are derived by means of the Cowan code RCG with the parameter set reported in Table IX. In the configuration notations, $A$ stands for $4 f^{11}, B$ for $4 f^{10}, d s^{2}$ for $5 d 6 s^{2}$, and $s p$ for $6 s 6 p$. Lowercase letters or Arabic numbers in the seventh column correspond to different possible parent terms [49]. Terms in parentheses are associated with the core configuration $A$ or $B$.

\begin{tabular}{|c|c|c|c|c|c|c|}
\hline $\begin{array}{l}E^{\text {expt }} \\
\left(\mathrm{cm}^{-1}\right)\end{array}$ & $\begin{array}{c}E^{\mathrm{th}} \\
\left(\mathrm{cm}^{-1}\right)\end{array}$ & $\begin{array}{c}\Delta E \\
\left(\mathrm{~cm}^{-1}\right)\end{array}$ & $g_{L}^{\text {th }}$ & $\begin{array}{c}\text { Leading } \\
\text { configuration }\end{array}$ & $\%$ & $\begin{array}{l}\text { Leading } \\
\text { LS term }\end{array}$ \\
\hline \multicolumn{7}{|c|}{$J=7 / 2$} \\
\hline 32758.37 & 32750.2 & 8 & 1.253 & $B-d s^{2}$ & 15 & $B-d s^{2}\left({ }^{5} S\right)^{4} D$ \\
\hline 32931.51 & 32935.7 & -4 & 1.232 & $B-d s^{2}$ & 25 & $B-d s^{2}\left({ }^{5} F\right)^{4} G$ \\
\hline 33188.42 & 33202.1 & -14 & 0.926 & $B-d s^{2}$ & 37 & $B-d s^{2}\left({ }^{5} F\right)^{4} H$ \\
\hline 35078.45 & 35105.5 & -27 & 1.147 & $B-d s^{2}$ & 19 & $B-d s^{2}\left({ }^{5} G\right)^{6} D$ \\
\hline 36001.87 & 35991.9 & 10 & 1.074 & $B-d s^{2}$ & 13 & $B-d s^{2}\left({ }^{5} G\right)^{6} G$ \\
\hline 36504.15 & \multicolumn{6}{|c|}{$J=9 / 2$} \\
\hline 16719.62 & 16727.3 & -8 & 1.118 & $B-d s^{2}$ & 40 & $B-d s^{2}\left({ }^{5} I\right)^{6} G$ \\
\hline 18757.87 & 18682.1 & 76 & 0.991 & $B-d s^{2}$ & 47 & $B-d s^{2}\left({ }^{5} I\right)^{6} I$ \\
\hline 21373.01 & 21371.6 & 1 & 1.140 & $B-d s^{2}$ & 62 & $B-d s^{2}\left({ }^{5} I\right)^{4} G$ \\
\hline 23861.17 & 23909.6 & -48 & 0.980 & $A-s p$ & 32 & $A-s p\left({ }^{4} I\right)^{6} H$ \\
\hline 24355.64 & 24367.3 & -12 & 1.137 & $B-d s^{2}$ & 27 & $B-d s^{2}\left({ }^{5} I\right)^{4} H$ \\
\hline 25453.49 & 25427.1 & 26 & 0.906 & $A-s p$ & 30 & $A-\operatorname{sp}\left({ }^{4} I\right)^{6} I$ \\
\hline 26039.99 & 25991.7 & 48 & 0.831 & $B-d s^{2}$ & 48 & $B-d s^{2}\left({ }^{5} I\right)^{4} I$ \\
\hline 28638.41 & 28653.1 & -15 & 1.456 & $B-d s^{2}$ & 54 & $B-d s^{2}\left({ }^{5} S\right)^{6} D$ \\
\hline 32039.69 & 32053.7 & -14 & 1.247 & $B-d s^{2}$ & 20 & $B-d s^{2}\left({ }^{5} G\right)^{6} D$ \\
\hline 33577.20 & 33553.1 & 24 & 1.159 & $B-d s^{2}$ & 18 & $B-d s^{2}\left({ }^{5} F\right)^{4} G$ \\
\hline \multicolumn{7}{|c|}{$J=11 / 2$} \\
\hline 13082.93 & 13094.9 & -12 & 1.260 & $B-d s^{2}$ & 50 & $B-d s^{2}\left({ }^{5} I\right)^{6} G$ \\
\hline 15792.13 & 15805.2 & -13 & 1.143 & $B-d s^{2}$ & 37 & $B-d s^{2}\left({ }^{5} I\right)^{6} I$ \\
\hline 16937.43 & 16958.5 & -21 & 1.244 & $B-d s^{2}$ & 52 & $B-d s^{2}\left({ }^{5} I\right)^{4} G$ \\
\hline 18491.21 & 18465.0 & 26 & 1.107 & $B-d s^{2}$ & 25 & $B-d s^{2}\left({ }^{5} I\right)^{6} H$ \\
\hline 18821.25 & 18802.1 & 19 & 1.107 & $A-s p$ & 46 & $A-s p\left({ }^{4} I\right)^{2} H$ \\
\hline 20493.40 & 20427.2 & 66 & 0.903 & $B-d s^{2}$ & 56 & $B-d s^{2}\left({ }^{5} I\right)^{6} K$ \\
\hline 20849.13 & 20863.0 & -14 & 1.104 & $B-d s^{2}$ & 32 & $B-d s^{2}\left({ }^{5} I\right)^{4} H$ \\
\hline 22413.14 & 22378.1 & 35 & 1.078 & $A-s p$ & 29 & $A-\operatorname{sp}\left({ }^{4} I\right)^{6} H$ \\
\hline 23379.31 & 23361.4 & 18 & 1.065 & $B-d s^{2}$ & 29 & $B-d s^{2}\left({ }^{5} I\right)^{4} I$ \\
\hline 23946.16 & 23961.3 & -15 & 1.057 & $A-s p$ & 30 & $A-s p\left({ }^{4} I\right)^{6} H$ \\
\hline 24141.21 & 24179.4 & -38 & 1.276 & $B-d s^{2}$ & 37 & $B-d s^{2}\left({ }^{5} F\right)^{6} F$ \\
\hline 24830.43 & 24907.2 & -77 & 0.968 & $A-s p$ & 27 & $A-s p\left({ }^{4} I\right)^{6} K$ \\
\hline 25261.55 & 25271.4 & -10 & 0.864 & $B-d s^{2}$ & 44 & $B-d s^{2}\left({ }^{5} I\right)^{4} K$ \\
\hline 25503.33 & 25467.7 & 36 & 0.998 & $A-s p$ & 14 & $A-\operatorname{sp}\left({ }^{4} I\right)^{6} K$ \\
\hline 25914.31 & 25997.5 & -83 & 1.209 & $B-d s^{2}$ & 32 & $B-d s^{2}\left({ }^{5} F\right)^{6} H$ \\
\hline 28793.03 & 28824.0 & -31 & 1.230 & $B-d s^{2}$ & 48 & $B-d s^{2}\left({ }^{5} F\right)^{4} G$ \\
\hline 29069.78 & 29016.7 & 53 & 1.154 & $A-s p$ & 33 & $A-s p\left({ }^{4} I\right)^{4} H b$ \\
\hline 29096.77 & 29132.9 & -36 & 1.078 & $A-s p$ & 19 & $A-\operatorname{sp}\left({ }^{4} F\right)^{6} F$ \\
\hline 30423.60 & 30332.7 & 91 & 1.240 & $B-d s^{2}$ & 39 & $B-d s^{2}\left({ }^{5} F\right)^{6} G$ \\
\hline 31903.28 & 31862.7 & 41 & 1.227 & $B-d s^{2}$ & 18 & $B-d s^{2}\left({ }^{5} F\right)^{6} G$ \\
\hline 32837.21 & 32860.6 & -23 & 1.014 & $A-s p$ & 31 & $A-s p\left({ }^{4} I\right)^{4} I b$ \\
\hline 33212.51 & 33287.1 & -75 & 1.206 & $A-s p$ & 23 & $A-\operatorname{sp}\left({ }^{2} H\right)^{4} G 2$ \\
\hline 33986.71 & 33965.6 & 21 & 1.104 & $A-s p$ & 10 & $A-\operatorname{sp}\left({ }^{2} H\right)^{2} I 2$ \\
\hline 34270.67 & 34292.7 & -22 & $\begin{array}{c}1.141 \\
J=\end{array}$ & $B-d s^{2}$ & 19 & $B-d s^{2}\left({ }^{5} G\right)^{4} G$ \\
\hline 9147.08 & 9117.3 & 30 & 1.338 & $B-d s^{2}$ & 66 & $B-d s^{2}\left({ }^{5} I\right)^{6} G$ \\
\hline 12344.55 & 12364.8 & -20 & 1.236 & $B-d s^{2}$ & 27 & $B-d s^{2}\left({ }^{5} I\right)^{6} I$ \\
\hline 15081.12 & 15112.6 & -32 & 1.177 & $B-d s^{2}$ & 46 & $B-d s^{2}\left({ }^{5} I\right)^{4} H$ \\
\hline 16735.95 & 16763.6 & -28 & 1.200 & $B-d s^{2}$ & 41 & $B-d s^{2}\left({ }^{5} I\right)^{6} H$ \\
\hline 17059.35 & 17019.9 & 39 & 1.193 & $A-s p$ & 29 & $A-\operatorname{sp}\left({ }^{4} I\right)^{4} H a$ \\
\hline 18564.90 & 18493.2 & 72 & 1.050 & $B-d s^{2}$ & 45 & $B-d s^{2}\left({ }^{5} I\right)^{6} K$ \\
\hline 18858.19 & 18792.4 & 66 & 1.138 & $A-s p$ & 37 & $A-\operatorname{sp}\left({ }^{4} I\right)^{2} I$ \\
\hline 20241.31 & 20266.4 & -25 & 1.104 & $B-d s^{2}$ & 43 & $B-d s^{2}\left({ }^{5} I\right)^{4} I$ \\
\hline
\end{tabular}


TABLE VIII. (Continued.)

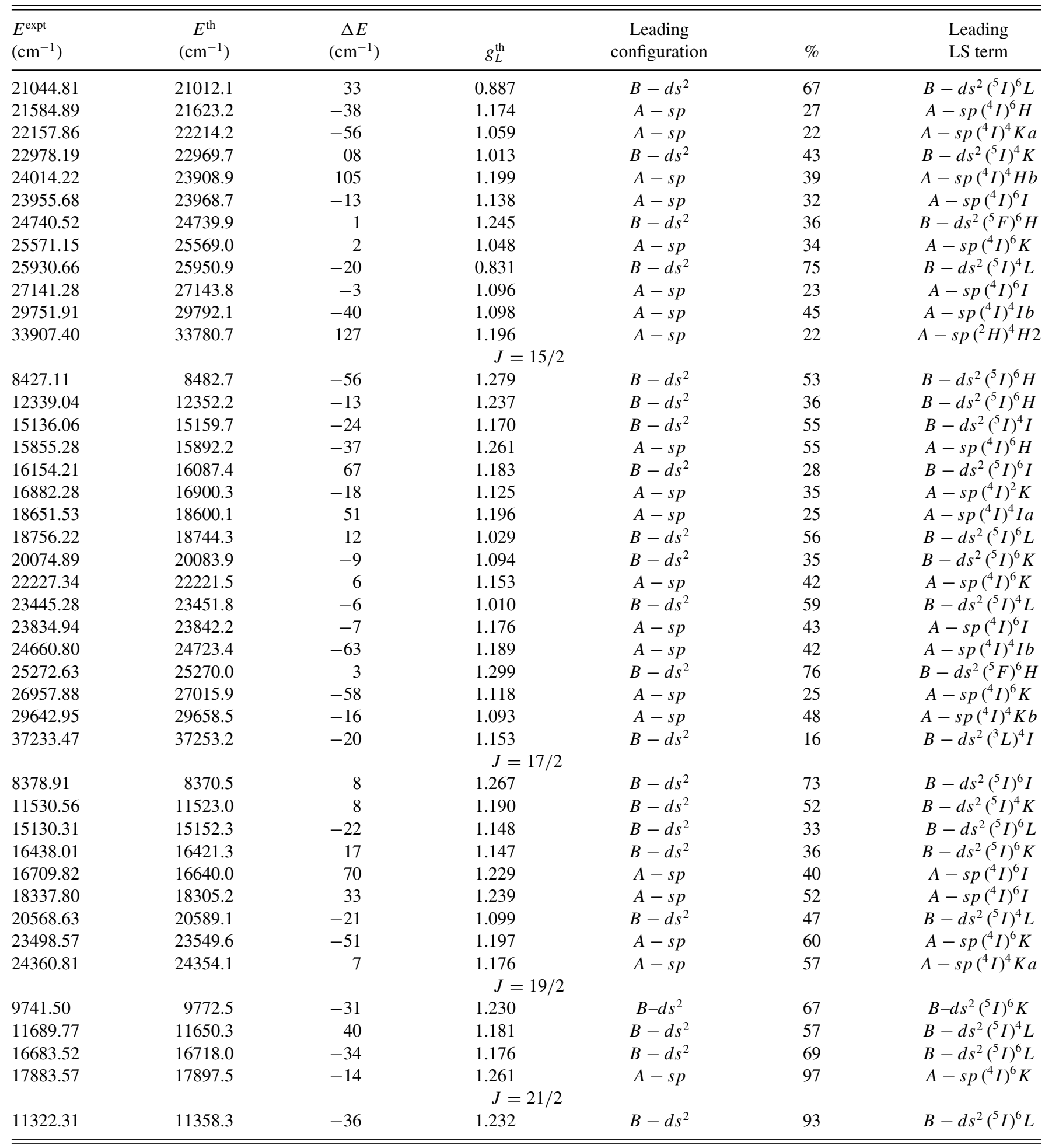

configuration-interaction mixing is stronger in erbium. This is very surprising, as the density of levels of dysprosium is higher around $25000 \mathrm{~cm}^{-1}$ and, so, a priori more favorable to configuration interaction. Again, the case of thulium will be particularly enlightening, because the large number of relatively strong transitions (with probabilities higher than $10^{7} \mathrm{~s}^{-1}$; see Ref. [58]) suggests even stronger configuration interaction than in erbium. Finally, we would like to emphasize that experimental measurements of heating rate or trap lifetimes are particularly welcome, in order to check the validity of our predictions.

\section{ACKNOWLEDGMENTS}

The authors acknowledge support from "DIM Nano-K" under the project "InterDy", and from Agence Nationale de la Recherche (ANR) under the project "COPOMOL" (Contract No. ANR-13-IS04-0004-01). 


\section{APPENDIX: EVEN-PARITY ENERGY LEVELS}

This Appendix presents the detailed calculations of holmium even-parity levels. Table VIII reports the results of our calculations, including the discrepancy between theoretical and experimental energies. Table IX lists the optimal parameters after the least-squares fitting procedure on energies.

TABLE IX. Fitted parameters (in $\mathrm{cm}^{-1}$ ) for even-parity configurations of Ho I compared with relativistic Hartree-Fock (HFR) values. The scaling factors are $\mathrm{SF}(P)=P_{\mathrm{fit}} / P_{\mathrm{HFR}}$, except for $E_{\mathrm{av}}$, where they equal $P_{\mathrm{fit}}-P_{\mathrm{HFR}}$. Some parameters are constrained to vary at a constant ratio $r_{n}$, indicated in the second column except when "fix" appears in the second or in the "Uncertainty" column. In this case, the parameter $P$ is not adjusted. The Uncertainty columns list the standard error of each parameter after the fitting procedure.

\begin{tabular}{|c|c|c|c|c|c|c|c|c|c|}
\hline \multirow{2}{*}{$\begin{array}{l}\text { Parameter } \\
P\end{array}$} & \multirow{2}{*}{$\begin{array}{c}\text { Constant } \\
\text { ratio }\end{array}$} & \multicolumn{4}{|c|}{ Fitted parameters } & \multicolumn{4}{|c|}{ Fitted parameters } \\
\hline & & $P_{\text {fit }}$ & Uncertainty & $P_{\mathrm{HFR}}$ & SF & $P_{\text {fit }}$ & Uncertainty & $P_{\mathrm{HFR}}$ & SF \\
\hline$E_{\mathrm{av}}$ & & 59617 & 105 & 5940 & 53677 & 51079 & 64 & 15134 & 35945 \\
\hline$F^{2}(4 f 4 f)$ & $r_{1}$ & 94927 & 707 & 125792 & 0.755 & 89432 & 666 & 118509 & 0.755 \\
\hline$F^{4}(4 f 4 f)$ & $r_{2}$ & 66088 & 1446 & 78881 & 0.838 & 61977 & 1356 & 73975 & 0.838 \\
\hline$F^{6}(4 f 4 f)$ & $r_{3}$ & 48377 & 1350 & 56738 & 0.853 & 45289 & 1264 & 53115 & 0.853 \\
\hline$\alpha$ & $r_{4}$ & 23.0 & 4 & & & 23.0 & 4 & & \\
\hline$\beta$ & Fix & -650 & & & & -650 & & & \\
\hline$\gamma$ & Fix & 2000 & & & & 2000 & & & \\
\hline$\zeta_{4 f}$ & $r_{5}$ & 2141 & 4 & 2193 & 0.976 & 2009 & 4 & 2058 & 0.976 \\
\hline$\zeta_{5 d}$ & $r_{7}$ & 757 & 11 & 920 & 0.823 & & & & \\
\hline$\zeta_{6 p}$ & $r_{16}$ & & & & & 1435 & 15 & 990 & 1.449 \\
\hline$F^{1}(4 f 5 d)$ & $r_{8}$ & 674 & 91 & & & & & & \\
\hline$F^{2}(4 f 5 d)$ & $r_{9}$ & 15491 & 279 & 20639 & 0.751 & & & & \\
\hline$F^{4}(4 f 5 d)$ & $r_{10}$ & 10954 & 464 & 9423 & 1.162 & & & & \\
\hline$F^{1}(4 f 6 p)$ & Fix & & & & & 150 & & & \\
\hline$F^{2}(4 f 6 p)$ & $r_{17}$ & & & & & 3643 & 289 & 3324 & 1.096 \\
\hline$G^{1}(4 f 5 d)$ & $r_{11}$ & 5410 & 151 & 8944 & 0.605 & & & & \\
\hline$G^{2}(4 f 5 d)$ & $r_{12}$ & 1378 & 434 & & & & & & \\
\hline$G^{3}(4 f 5 d)$ & $r_{13}$ & 6036 & 460 & 7086 & 0.852 & & & & \\
\hline$G^{4}(4 f 5 d)$ & $r_{14}$ & 2314 & 546 & & & & & & \\
\hline$G^{5}(4 f 5 d)$ & $r_{15}$ & 4508 & 306 & 5353 & 0.842 & & & & \\
\hline$G^{3}(4 f 6 s)$ & $r_{18}$ & & & & & 1358 & 92 & 1676 & 0.810 \\
\hline$G^{2}(4 f 6 p)$ & Fix & & & & & 760 & & 760 & 1.0 \\
\hline$G^{4}(4 f 6 p)$ & Fix & & & & & 662 & & 662 & 1.0 \\
\hline$G^{1}(6 s 6 p)$ & $r_{19}$ & & & & & 10321 & 74 & 23282 & 0.443 \\
\hline Configuration interaction & & & $4 f^{10} 5 d 6 s^{2}-$ & $f^{11} 6 s 6 p$ & & & & & \\
\hline$R^{1}(5 d 6 s, 4 f 6 p)$ & $r_{6}$ & -3223 & 150 & -4555 & 0.708 & & & & \\
\hline$R^{3}(5 d 6 s, 6 p 4 f)$ & $r_{6}$ & -685 & 32 & -968 & 0.708 & & & & \\
\hline
\end{tabular}

[1] A. Frisch, M. Mark, K. Aikawa, F. Ferlaino, J. L. Bohn, C. Makrides, A. Petrov, and S. Kotochigova, Quantum chaos in ultracold collisions of gas-phase erbium atoms, Nature 507, 475 (2014).

[2] T. Maier, H. Kadau, M. Schmitt, M. Wenzel, I. Ferrier-Barbut, T. Pfau, A. Frisch, S. Baier, K. Aikawa, L. Chomaz, M. J. Mark, F. Ferlaino, C. Makrides, E. Tiesinga, A. Petrov, and S. Kotochigova, Emergence of Chaotic Scattering in Ultracold Er and Dy, Phys. Rev. X 5, 041029 (2015).

[3] Y. Tang, A. Sykes, N. Q. Burdick, J. L. Bohn, and B. L. Lev, $s$-wave scattering lengths of the strongly dipolar bosons ${ }^{162} \mathrm{Dy}$ and ${ }^{160}$ Dy, Phys. Rev. A 92, 022703 (2015).

[4] M. Lu, N. Q. Burdick, and B. L. Lev, Quantum Degenerate Dipolar Fermi Gas, Phys. Rev. Lett. 108, 215301 (2012).

[5] N. Nessi, A. Iucci, and M. A. Cazalilla, Quantum Quench and Prethermalization Dynamics in a Two-Dimensional Fermi Gas with Long-Range Interactions, Phys. Rev. Lett. 113, 210402 (2014).
[6] A. Frisch, M. Mark, K. Aikawa, S. Baier, R. Grimm, A. Petrov, S. Kotochigova, G. Quéméner, M. Lepers, O. Dulieu, and F. Ferlaino, Ultracold Polar Molecules Composed of Strongly Magnetic Atoms, Phys. Rev. Lett. 115, 203201 (2015).

[7] N. Y. Yao, S. D. Bennett, C. R. Laumann, B. L. Lev, and A. V. Gorshkov, Bilayer fractional quantum Hall states with dipoles, Phys. Rev. A 92, 033609 (2015).

[8] S. Baier, M. J. Mark, D. Petter, K. Aikawa, L. Chomaz, Z. Cai, M. Baranov, P. Zoller, and F. Ferlaino, Extended Bose-Hubbard models with ultracold magnetic atoms, Science 352, 201 (2016).

[9] T. Maier, I. Ferrier-Barbut, H. Kadau, M. Schmitt, M. Wenzel, C. Wink, T. Pfau, K. Jachymski, and P. S. Julienne, Broad universal Feshbach resonances in the chaotic spectrum of dysprosium atoms, Phys. Rev. A 92, 060702 (2015).

[10] N. Q. Burdick, K. Baumann, Y. Tang, M. Lu, and B. L. Lev, Fermionic Suppression of Dipolar Relaxation, Phys. Rev. Lett. 114, 023201 (2015). 
[11] B. M. Fregoso, K. Sun, E. Fradkin, and B. L. Lev, Biaxial nematic phases in ultracold dipolar Fermi gases, New J. Phys. 11, 103003 (2009).

[12] K. Aikawa, S. Baier, A. Frisch, M. Mark, C. Ravensbergen, and F. Ferlaino, Observation of Fermi surface deformation in a dipolar quantum gas, Science 345, 1484 (2014).

[13] H. Kadau, M. Schmitt, M. Wenzel, C. Wink, T. Maier, I. FerrierBarbut, and T. Pfau, Observing the Rosensweig instability of a quantum ferrofluid, Nature 530, 194 (2016).

[14] I. Ferrier-Barbut, H. Kadau, M. Schmitt, M. Wenzel, and T. Pfau, Observation of Quantum Droplets in a Strongly Dipolar Bose Gas, Phys. Rev. Lett. 116, 215301 (2016).

[15] K.-T. Xi and H. Saito, Droplet formation in a Bose-Einstein condensate with strong dipole-dipole interaction, Phys. Rev. A 93, 011604 (2016).

[16] A. Macia, J. Sánchez-Baena, J. Boronat, and F. Mazzanti, Droplets of Trapped Quantum Dipolar Bosons, Phys. Rev. Lett. 117, 205301 (2016).

[17] X. Cui, B. Lian, T.-L. Ho, B. L. Lev, and H. Zhai, Synthetic gauge field with highly magnetic lanthanide atoms, Phys. Rev. A 88, 011601 (2013).

[18] N. Q. Burdick, Y. Tang, and B. L. Lev, A Long-Lived SpinOrbit-Coupled Degenerate Dipolar Fermi Gas, Phys. Rev. X 6, 031022 (2016).

[19] A. Kozlov, V. A. Dzuba, and V. V. Flambaum, Prospects of building optical atomic clocks using Er I or Er III, Phys. Rev. A 88, 032509 (2013).

[20] G. A. Vishnyakova, E. S. Kalganova, D. D. Sukachev, S. A. Fedorov, A. V. Sokolov, A. V. Akimov, N. N. Kolachevsky, and V. N. Sorokin, Two-stage laser cooling and optical trapping of thulium atoms, Laser Phys. 24, 074018 (2014).

[21] D. Sukachev, S. Fedorov, I. Tolstikhina, E. Kalganova, G. Vishnyakova, K. Khabarova, D. Tregubov, A. Golovizin, V. Sorokin, and N. Kolachevsky, Inner-shell magnetic dipole transition in Tm atoms as a candidate for optical lattice clocks, Phys. Rev. A 94, 022512 (2016).

[22] C. I. Hancox, S. C. Doret, M. T. Hummon, L. Luo, and J. M. Doyle, Magnetic trapping of rare-earth atoms at millikelvin temperatures, Nature 431, 281 (2004).

[23] B. Hemmerling, G. K. Drayna, E. Chae, A. Ravi, and J. M. Doyle, Buffer gas loaded magneto-optical traps for $\mathrm{Yb}, \mathrm{Tm}, \mathrm{Er}$ and Ho, New J. Phys. 16, 063070 (2014).

[24] N. Leefer, A. Cingöz, B. Gerber-Siff, A. Sharma, J. R. Torgerson, and D. Budker, Transverse laser cooling of a thermal atomic beam of dysprosium, Phys. Rev. A 81, 043427 (2010).

[25] M. Lu, S. H. Youn, and B. L. Lev, Trapping Ultracold Dysprosium: A Highly Magnetic Gas for Dipolar Physics, Phys. Rev. Lett. 104, 063001 (2010).

[26] T. Maier, H. Kadau, M. Schmitt, A. Griesmaier, and T. Pfau, Narrow-line magneto-optical trap for dysprosium atoms, Opt. Lett. 39, 3138 (2014).

[27] D. Dreon, L. A. Sidorenkov, C. Bouazza, W. Maineult, J. Dalibard, and S. Nascimbene, Optical cooling and trapping of highly magnetic atoms: The benefits of a spontaneous spin polarization, J. Phys. B 50, 065005 (2017).

[28] H. Ban, M. Jacka, J. Hanssen, J. Reader, and J. McClelland, Laser cooling transitions in atomic erbium, Opt. Express 13, 3185 (2005).
[29] J. J. McClelland and J. L. Hanssen, Laser Cooling Without Repumping: A Magneto-Optical Trap for Erbium Atoms, Phys. Rev. Lett. 96, 143005 (2006).

[30] A. Frisch, K. Aikawa, M. Mark, A. Rietzler, J. Schindler, E. Zupanič, R. Grimm, and F. Ferlaino, Narrow-line magnetooptical trap for erbium, Phys. Rev. A 85, 051401 (2012).

[31] J. Miao, J. Hostetter, G. Stratis, and M. Saffman, Magnetooptical trapping of holmium atoms, Phys. Rev. A 89, 041401 (2014).

[32] D. Sukachev, A. Sokolov, K. Chebakov, A. Akimov, S. Kanorsky, N. Kolachevsky, and V. Sorokin, Magneto-optical trap for thulium atoms, Phys. Rev. A 82, 011405 (2010).

[33] M. Lu, N. Q. Burdick, S. H. Youn, and B. L. Lev, Strongly Dipolar Bose-Einstein Condensate of Dysprosium, Phys. Rev. Lett. 107, 190401 (2011).

[34] Y. Tang, N. Q. Burdick, K. Baumann, and B. L. Lev, BoseEinstein condensation of ${ }^{162}$ Dy and ${ }^{160}$ Dy, New J. Phys. 17, 045006 (2015).

[35] K. Aikawa, A. Frisch, M. Mark, S. Baier, A. Rietzler, R. Grimm, and F. Ferlaino, Bose-Einstein Condensation of Erbium, Phys. Rev. Lett. 108, 210401 (2012).

[36] K. Aikawa, A. Frisch, M. Mark, S. Baier, R. Grimm, and F. Ferlaino, Reaching Fermi Degeneracy via Universal Dipolar Scattering, Phys. Rev. Lett. 112, 010404 (2014).

[37] M. Saffman and K. Mølmer, Scaling the neutral-atom Rydberg gate quantum computer by collective encoding in holmium atoms, Phys. Rev. A 78, 012336 (2008).

[38] J. Hostetter, J. D. Pritchard, J. E. Lawler, and M. Saffman, Measurement of holmium Rydberg series through magnetooptical trap depletion spectroscopy, Phys. Rev. A 91, 012507 (2015).

[39] B. K. Newman, N. Brahms, Y. S. Au, C. Johnson, C. B. Connolly, J. M. Doyle, D. Kleppner, and T. J. Greytak, Magnetic relaxation in dysprosium-dysprosium collisions, Phys. Rev. A 83, 012713 (2011).

[40] W. Kao, Y. Tang, N. Q. Burdick, and B. L. Lev, Anisotropic dependence of tune-out wavelength near the Dy 741-nm transition, Optics Express 25, 3411 (2017).

[41] T. Miyamachi, T. Schuh, T. Märkl, C. Bresch, T. Balashov, A. Stöhr, C. Karlewski, S. André, M. Marthaler, M. Hoffmann et al., Stabilizing the magnetic moment of single holmium atoms by symmetry, Nature 503, 242 (2013).

[42] M. Shiddiq, D. Komijani, Y. Duan, A. Gaita-Ariño, E. Coronado, and S. Hill, Enhancing coherence in molecular spin qubits via atomic clock transitions, Nature 531, 348 (2016).

[43] N. L. Manakov, V. D. Ovsiannikov, and L. P. Rapoport, Atoms in a laser field, Phys. Rep. 141, 320 (1986).

[44] R. Grimm, M. Weidemüller, and Y. B. Ovchinnikov, Optical dipole traps for neutral atoms, Adv. At. Mol. Opt. Phys. 42, 95 (2000).

[45] M. Lepers, J.-F. Wyart, and O. Dulieu, Anisotropic optical trapping of ultracold erbium atoms, Phys. Rev. A 89, 022505 (2014).

[46] H. Li, J.-F. Wyart, O. Dulieu, S. Nascimbène, and M. Lepers, Optical trapping of ultracold dysprosium atoms: Transition probabilities, dynamic dipole polarizabilities and van der Waals $C_{6}$ coefficients, J. Phys. B 50, 014005 (2017).

[47] J.-F. Wyart, On the interpretation of complex atomic spectra by means of the parametric Racah-Slater method and Cowan codes, Can. J. Phys. 89, 451 (2011). 
[48] M. Lepers, Y. Hong, J.-F. Wyart, and O. Dulieu, Proposal for laser cooling of rare-earth ions, Phys. Rev. A 93, 011401(R) (2016).

[49] R. D. Cowan, The Theory of Atomic Structure and Spectra, Vol. 3 (University of California Press, Berkeley, 1981).

[50] K. Beloy, Theory of the ac stark effect on the atomic hyperfine structure and applications to microwave atomic clocks, Ph.D. thesis, University of Nevada, Reno, 2009.

[51] P. W. Langhoff, S. T. Epstein, and M. Karplus, Aspects of time-dependent perturbation theory, Rev. Mod. Phys. 44, 602 (1972).

[52] D. A. Varshalovich, A. N. Moskalev, and V. K. Khersonskii, Quantum Theory of Angular Momentum (World Scientific, Singapore, 1988).

[53] J. R. P. Angel and P. G. H. Sandars, The hyperfine structure Stark effect. I. Theory, Proc. R. Soc. London A 305, 125 (1968).

[54] A. Kramida, Yu. Ralchenko, J. Reader, and NIST ASD Team, NIST Atomic Spectra Database (Version 5.3) (National Institute of Standards and Technology, Gaithersburg, MD, 2015). Available at: http://physics.nist.gov/asd.

[55] M. E. Wickliffe, J. E. Lawler, and G. Nave, Atomic transition probabilities for Dy I and Dy II, J. Quant. Spectrosc. Radiat. Transfer 66, 363 (2000).

[56] G. Nave, Atomic transition rates for neutral holmium (Ho I), J. Opt. Soc. Am. B 20, 2193 (2003).

[57] J. E. Lawler, J. F. Wyart, and E. A. Den Hartog, Atomic transition probabilities of Er I, J. Phys. B 43, 235001 (2010).

[58] M. E. Wickliffe and J. E. Lawler, Atomic transition probabilities for Tm I and Tm II, J. Opt. Soc. Am. B 14, 737 (1997).

[59] W. C. Martin, R. Zalubas, and L. Hagan, Atomic energy levelsthe rare-earth elements, Stand. Ref. Data Ser. (Natl. Bur. Stand., U.S.) 60, 332 (1978).

[60] S. Kröger, J.-F. Wyart, and P. Luc, Theoretical interpretation of hyperfine structures in doubly-excited configurations
$4 f 105 d 6 s 6 p$ and $4 f 105 d 26 s$ and new energy levels in neutral holmium (Ho I), Phys. Scripta 55, 579 (1997).

[61] J.-F. Wyart, An interpretation of Dy I and Ho I energy levels in the ground state parity (unpublished).

[62] L. Brewer, in Systematics of the Properties of the Lanthanides, edited by S. P. Sinha and D. Reidel (Dordrecht, Netherlands, 1983).

[63] J.-F. Wyart, Interpretation du spectre de Dy I: I. Etude des configurations impaires, Physica 75, 371 (1974).

[64] L. Ma, J. Indergaard, B. Zhang, I. Larkin, R. Moro, and W. A. de Heer, Measured atomic ground-state polarizabilities of 35 metallic elements, Phys. Rev. A 91, 010501(R) (2015).

[65] D. R. Lide, CRC Handbook of Chemistry and Physics (CRC Press, Boca Raton, FL, 2012).

[66] X. Chu, A. Dalgarno, and G. C. Groenenboom, Dynamic polarizabilities of rare-earth-metal atoms and dispersion coefficients for their interaction with helium atoms, Phys. Rev. A 75, 032723 (2007).

[67] V. A. Dzuba, A. Kozlov, and V. V. Flambaum, Scalar static polarizabilities of lanthanides and actinides, Phys. Rev. A 89, 042507 (2014).

[68] V. A. Dzuba, Ionization potentials and polarizabilities of superheavy elements from $\mathrm{Db}$ to $\mathrm{Cn}(z=105-112)$, Phys. Rev. A 93, 032519 (2016).

[69] R.-H. Rinkleff and F. Thorn, On the tensor polarizabilities in the $4 f^{n} 6 s^{2}$ ground levels of the neutral rare-earth atoms, Z. Phys. D 32, 173 (1994).

[70] A. J. Stone, The Theory of Intermolecular Forces (Oxford University Press, New York, 1996).

[71] I. G. Kaplan, Intermolecular Interactions: Physical Picture, Computational Methods and Model Potentials (John Wiley \& Sons, New York, 2006).

[72] M. Lepers and O. Dulieu, Long-range interactions between ultracold atoms and molecules, arXiv:1703.02833. 